\title{
Modelling diesel engines with a variable- geometry turbocharger and exhaust gas recirculation by optimization of model parameters for capturing non-linear system dynamics
}

Johan Wahlström and Lars Eriksson

\author{
Linköping University Post Print
}

N.B.: When citing this work, cite the original article.

Original Publication:

Johan Wahlström and Lars Eriksson, Modelling diesel engines with a variable-geometry turbocharger and exhaust gas recirculation by optimization of model parameters for capturing non-linear system dynamics, 2011, Proceedings of the Institution of mechanical engineers. Part D, journal of automobile engineering, (225), 7, 960-986.

http://dx.doi.org/10.1177/0954407011398177

Copyright: Professional Engineering Publishing (Institution of Mechanical Engineers) http://journals.pepublishing.com/

Postprint available at: Linköping University Electronic Press http://urn.kb.se/resolve?urn=urn:nbn:se:liu:diva-69615 


\title{
Modelling diesel engines with a variable-geometry turbocharger and exhaust gas recirculation by optimization of model parameters for capturing non-linear system dynamics
}

\author{
J Wahlström* and L Eriksson \\ Department of Electrical Engineering, Linköping University, Linköping, Sweden
}

The manuscript was received on 12 February 2010 and was accepted after revision for publication on 4 January 2011.

DOI: $\quad 10.1177 / 0954407011398177$

\begin{abstract}
A mean-value model of a diesel engine with a variable-geometry turbocharger (VGT) and exhaust gas recirculation (EGR) is developed, parameterized, and validated. The intended model applications are system analysis, simulation, and development of modelbased control systems. The goal is to construct a model that describes the gas flow dynamics including the dynamics in the manifold pressures, turbocharger, EGR, and actuators with few states in order to obtain short simulation times. An investigation of model complexity and descriptive capabilities is performed, resulting in a model that has only eight states. A Simulink implementation including a complete set of parameters of the model are available for download. To tune and validate the model, stationary and dynamic measurements have been performed in an engine laboratory. All the model parameters are estimated automatically using weighted least-squares optimization and it is shown that it is important to tune both the submodels and the complete model and not only the submodels or not only the complete model. In static and dynamic validations of the entire model, it is shown that the mean relative errors are 5.8 per cent or lower for all measured variables. The validations also show that the proposed model captures the system properties that are important for control design, i.e. a non-minimum phase behaviour in the channel EGR valve to the intake manifold pressure and a non-minimum phase behaviour, an overshoot, and a sign reversal in the VGT to the compressor mass flow channel, as well as couplings between channels.
\end{abstract}

Keywords: diesel engines, modelling, variable-geometry turbocharger, exhaust gas recirculation, non-linear system

\section{INTRODUCTION}

Legislated emission limits for heavy-duty trucks are constantly being reduced. To fulfil the requirements, technologies such as exhaust gas recirculation (EGR) systems and variable-geometry turbochargers (VGTs) have been introduced. The primary emission reduction mechanisms utilized to control the emissions

*Corresponding author: Department of Electrical Engineering, Linköping University, Linköping 58183, Sweden.

email: johan.erik.wahlstrom@gmail.com are that nitrogen oxides $\mathrm{NO}_{x}$ can be reduced by increasing the intake manifold EGR fraction $x_{\text {egr }}$ and smoke can be reduced by increasing the oxygento-fuel ratio $\lambda_{\mathrm{O}}$ [1]. However, $x_{\text {egr }}$ and $\lambda_{\mathrm{O}}$ depend in complicated ways on the actuation of the EGR and VGT. It is therefore necessary to have coordinated control of the EGR and VGT to reach the legislated emission limits in $\mathrm{NO}_{x}$ and smoke. When developing and validating a controller for this system, it is desirable to have a model that describes the system dynamics and the non-linear effects that are important for gas flow control. These important properties 
have been described in references [2] to [4], showing that this system has non-minimum phase behaviours, overshoots, and sign reversals. Therefore, the objective of this paper is to construct a mean-value diesel engine model, from the actuator input to the system output, that captures these properties. The intended applications of the model are system analysis, simulation, and development of model-based control systems. The model will describe the dynamics in the manifold pressures, turbocharger, EGR, and actuators with few states in order to obtain short simulation times.

A successful modelling is the combined result of experience and systematic analysis of a problem and balances model complexity with descriptive capability. This paper collects and summarizes the knowledge gained over several years about models for EGR and/or VGT systems and presents a complete and validated model for such an engine. The main contributions in this paper are as follows.

1. An analysis is given of how model assumptions influence the system dynamics highlighting the balance between the model complexity and the dynamics.

2. It is shown that inclusion of the temperature states and the pressure drop over the intercooler have only minor effects on the dynamic behaviour and that they have a minor influence on the model quality.

3. Based on the analysis a model structure is proposed, where the emphasis is on using a small set of states. The essence of the system is captured by five states related to gas flows. In addition, actuator models are necessary in many applications and here the experimental results show that three states are needed.

4. A tuning method for the model parameters, consisting of initialization methods for each submodel and least-squares optimization, is described. In system identification and in tuning of mean-value engine models, it is common to use either the submodels or the complete model in this optimization. However, here it is shown that it is important to use both the submodels and the complete model in the optimization to reduce errors in the complete model.

5. The cylinder-out temperature is modelled using a fixed-point iteration and it is shown that it is sufficient to use one iteration in this iterative process to achieve an accuracy of 0.15 per cent.

6. A survey of diesel engine model components is provided, summarizing related work and its relation to the proposed model.
A key feature of the resulting model is its component-based structure with parameterized functions for describing the component performance. This reduces the dependence on maps and their associated performance. The model is implemented as a Simulink file complemented by a parameter file and is available as a companion to this paper [5]; this implementation is provided for download or by contacting the authors. More details about the provided model are given in Appendix 3. Further, the model reported here has received widespread acceptance in industry and is used in both industrial and academic applications (see, for example, references [6] to [15]).

\subsection{Outline}

A survey of different models is presented in section 2. This section also briefly describes the structure of the proposed model as well as the tuning and the validation. Model equations are described for each submodel in sections 3 to 7 . Section 8 describes the optimization of the model parameters and the model validation. The goal is also to investigate whether the proposed model can be improved with model extensions in section 9 .

\section{SURVEY AND MODEL SUMMARY}

The proposed model is a mean-value engine model that uses filling and emptying concepts for the manifolds $[1,16,17]$. Several mean-value models with different selections of states and complexities have been published for diesel engines with EGR and VGT. A third-order model that describes the intake and exhaust manifold pressure and turbocharger dynamics has been developed in reference [18]. The model in reference [3] has six states describing the intake and exhaust manifold pressures, the temperature dynamics, and the turbocharger and compressor mass flow dynamics. A seventh-order model that describes the intake and exhaust manifold pressure, temperature, air-mass fraction dynamics, and turbocharger dynamics was proposed in reference [19]. These dynamics are also described by the seventhorder models in references [2], [4], and [18], where the burned gas fraction is used instead of the airmass fraction in the manifolds. Another model that describes these dynamics is the ninth-order model in reference [20] which also has two states for the actuator dynamics.

The model proposed here has eight states: the intake manifold pressure $p_{\text {im }}$, the exhaust manifold pressures $p_{\mathrm{em}}$, the oxygen mass fraction $X_{\mathrm{Oim}}$ in the 


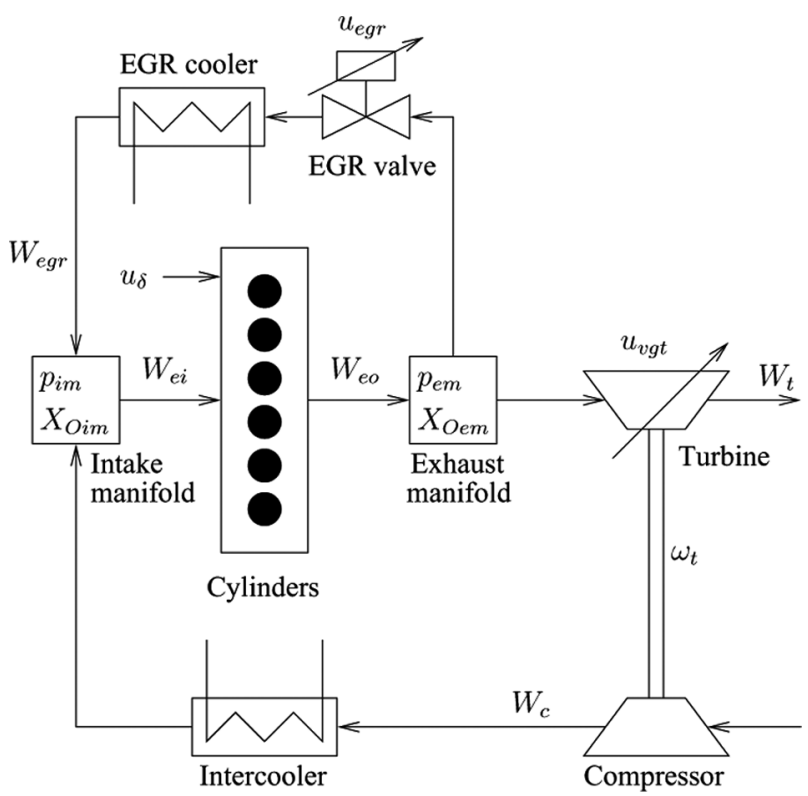

Fig. 1 A model structure of the diesel engine. It has three control inputs and five main states related to the engine $\left(p_{\mathrm{im}}, p_{\mathrm{em}}, X_{\mathrm{Oim}}, X_{\mathrm{Oem}}\right.$, and $\left.\omega_{\mathrm{t}}\right)$. In addition, there are three states for actuator dynamics $\left(\tilde{u}_{\text {egrl }}, \tilde{u}_{\text {egr2}}\right.$, and $\left.\tilde{u}_{\text {vgt }}\right)$

intake manifold, the oxygen mass fraction $X_{\text {Oem }}$ in the exhaust manifold, the turbocharger speed $\omega_{\mathrm{t}}$, and three states $\left(\tilde{u}_{\text {egr } 1}, \tilde{u}_{\text {egr2 }}\right.$, and $\left.\tilde{u}_{\text {vgt }}\right)$ describing the actuator dynamics for the two control signals. These states are collected in a state vector $\boldsymbol{x}$ according to

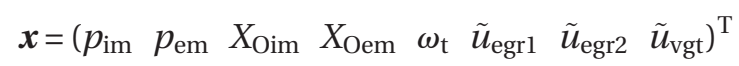

Descriptions of the notation and the variables can be found in the Appendices, and the structure of the model can be seen in Fig. 1.

The states $p_{\mathrm{im}}, p_{\mathrm{em}}$, and $\omega_{\mathrm{t}}$ describe the main dynamics and the most important system properties, such as the non-minimum phase behaviours, overshoots, and sign reversals. In order to model the dynamics in the oxygen-to-fuel ratio $\lambda_{\mathrm{O}}$, the states $X_{\text {Oim }}$ and $X_{\text {Oem }}$ are used. Finally, the states $\tilde{u}_{\text {egr1 }}, \tilde{u}_{\text {egr2 }}$, and $\tilde{u}_{\text {vgt }}$ describe the actuator dynamics where the EGR valve actuator model has two states $\tilde{u}_{\text {egr1 }}$ and $\tilde{u}_{\text {egr2 }}$ in order to describe an overshoot in the actuator.

Many models in the literature, which have approximately the same complexities as the model proposed here, use three states for each control volume in order to describe the temperature dynamics [2, 18, 20]. However, the model proposed here uses only two states for each manifold: pressure and oxygen mass fraction. Model extensions are investigated in section 9.1, showing that inclusion of the temperature states has only minor effects on the dynamic behaviour. Furthermore, the pressure drop over the intercooler is not modelled since this pressure drop has only small effects on the dynamic behaviour. However, this pressure drop has large effects on the stationary values, but these effects do not improve the complete engine model (see section 9.2).

\subsection{Model structure}

It is important that the model can be utilized both for different vehicles and for engine testing and calibration. In these situations the engine operation is defined by the rotational speed $n_{\mathrm{e}}$, e.g. given as an input from a drive cycle, and therefore it is natural to parameterize the model using the engine speed. The resulting model is thus expressed in state space form as

$$
\dot{\boldsymbol{x}}=f\left(\boldsymbol{x}, \boldsymbol{u}, n_{\mathrm{e}}\right)
$$

where the engine speed $n_{\mathrm{e}}$ is considered as a measured disturbance, and $\boldsymbol{u}$ is the control input vector given by

$$
\boldsymbol{u}=\left(\begin{array}{lll}
u_{\delta} & u_{\text {egr }} & u_{\text {vgt }}
\end{array}\right)^{\mathrm{T}}
$$

which contains a mass $u_{\delta}$ of injected fuel, EGR valve position $u_{\text {egr }}$, and VGT actuator position $u_{\text {vgt }}$. The EGR valve is closed when $u_{\text {egr }}=0$ per cent and open when $u_{\text {egr }}=100$ percent. The VGT is closed when $u_{\mathrm{vgt}}=0$ percent and open when $u_{\mathrm{vgt}}=100$ per cent.

\subsection{Measurements}

To tune and validate the model, stationary and dynamic measurements were performed in an engine laboratory at Scania $\mathrm{CV} \mathrm{AB}$, and these are described below. Furthermore, in Appendix 2 the sensor dynamics are investigated showing that the dynamics visible in the experimental data are due to the system dynamics, this is because the sensors are much faster than the system dynamics.

\subsubsection{Stationary measurements}

The stationary data consist of measurements in stationary conditions at 82 operating points, which are scattered over a large operating region covering different loads, speeds, and VGT and EGR positions. These 82 operating points also include the European stationary cycle at 13 operating points. The variables that were measured during stationary measurements are given later in Table 5 in Appendix 2. 
Table 1 Dynamic tuning and validation data that consist of steps in the VGT position, EGR valve, and fuel injection. The data sets B, C, I, and J are used for tuning of dynamic models, the data sets A to I are used for tuning the static models, the data sets A and D to I are used for validating the dynamic models, and the data sets $\mathrm{Q}$ to $\mathrm{V}$ are used for validating both the static and the dynamic models

\begin{tabular}{|c|c|c|c|c|c|c|c|c|c|c|c|c|c|c|c|c|}
\hline \multirow[b]{2}{*}{ Data set } & \multicolumn{13}{|c|}{ VGT and EGR steps } & \multicolumn{2}{|c|}{$u_{\delta}$ steps } & \multirow{2}{*}{$\begin{array}{l}\text { VGT and EGR steps } \\
\text { J }\end{array}$} \\
\hline & A & B & Q & $\mathrm{C}$ & $\mathrm{R}$ & $\mathrm{D}$ & $\mathrm{S}$ & $\mathrm{E}$ & $\mathrm{T}$ & $\mathrm{F}$ & $\mathrm{U}$ & G & $\mathrm{H}$ & I & $\mathrm{V}$ & \\
\hline Speed (r/min) & 1200 & 1200 & 1200 & 1200 & 1200 & 1200 & 1500 & 1500 & 1500 & 1900 & 1900 & 1900 & 1900 & 1500 & 1500 & - \\
\hline Load $(\%)$ & 25 & 40 & 40 & 50 & 50 & 75 & 25 & 50 & 75 & 25 & 50 & 75 & 100 & - & - & - \\
\hline Number of steps & 77 & 23 & 12 & 2 & 77 & 77 & 77 & 77 & 77 & 77 & 77 & 55 & 1 & 7 & 28 & 48 \\
\hline Sample frequency $(\mathrm{Hz})$ & 1 & 100 & 100 & 100 & 1 & 1 & 1 & 1 & 1 & 1 & 1 & 1 & 100 & 10 & 1 & 100 \\
\hline
\end{tabular}

All the stationary measurements are used for tuning the parameters in static models. The static models are then validated using dynamic measurements.

\subsubsection{Dynamic measurements}

The dynamic data consist of measurements in dynamic conditions with steps in the VGT control signal, EGR control signal, and fuel injection at several different operating points according to Table 1. The steps in the VGT position and EGR valve are performed for the data sets A to $\mathrm{H}, \mathrm{J}$, and Q to $\mathrm{U}$, and the steps in fuel injection are performed in the data sets I and V. The data set $\mathrm{J}$ is used for tuning the dynamic actuator models and the data sets B, C, and $\mathrm{I}$ are used for tuning the dynamic models in the manifolds, in the turbocharger, and in the engine torque respectively. Further, the data sets A to I are used for tuning the static models, the data sets $A$ and $D$ to I are used for validation of the dynamic models, and the data sets Q to $\mathrm{V}$ are used for validation of both the static and the dynamic models. The main difference between the tuning and validation data is that they have different engine loads and thus different flows and pressures.

The dynamic measurements are limited in the sample rate with a sample frequency of $1 \mathrm{~Hz}$ for the data sets $A, D$ to $G$, and $R$ to $V, 10 \mathrm{~Hz}$ for the data set I, and $100 \mathrm{~Hz}$ for the data sets $\mathrm{B}, \mathrm{C}, \mathrm{H}, \mathrm{J}$, and Q. This means that the data sets A, D to G, I, and R to $\mathrm{V}$, do not capture the fastest dynamics in the system, while the data sets $\mathrm{B}, \mathrm{C}, \mathrm{H}, \mathrm{J}$, and Q do. Further, the data sets B, C, H, J, and Q were measured 3.5 years after the data sets A, D to G, I and R to $\mathrm{V}$ and the stationary data. The variables that were measured during dynamic measurements can be seen later in Table 6 in Appendix 2 .

\subsection{Parameter estimation and validation}

The model parameters are estimated in five steps. First, the static parameters are initialized using least-squares optimization for each submodel in section 3 to 6 and data from stationary measurements. Second, the actuator parameters are estimated using least-squares optimization for the actuator models and using the dynamic measurements in the data set $\mathrm{J}$ in Table 1 . Third, the manifold volumes and the turbocharger inertia and, fourth, the time constant for the engine torque are estimated using least-squares optimization for the complete model and using data from the dynamic measurements in the data sets $\mathrm{B}, \mathrm{C}$, and $\mathrm{I}$ in Table 1. Fifth, the static parameters are finally estimated using least-squares optimization for each submodel and for the complete model and using data from both the stationary and the dynamic measurements. The dynamic measurements used are the data sets A to I in Table 1. In the fifth step the static parameters are initialized using the method in the first step.

Systematic tuning methods for each parameter are described in detail in sections 3 to 6 , and section 8.1. In section 8.2 it is shown that is important to use both the submodels and the complete model in the tuning method to reduce errors in the complete model. Since the proposed tuning methods are systematic and general, it is straightforward to re-create the values of the model parameters and to apply the tuning methods to different diesel engines with EGR and VGT.

Because there were only a few stationary measurements, both the static and the dynamic models are validated by simulating the complete model and comparing it with the dynamic measurements $\mathrm{Q}$ to $\mathrm{V}$ in Table 1. The dynamic models are also validated using the dynamic measurements A and D to I in Table. 1.

\subsection{Relative error}

Relative errors are calculated and used to evaluate the tuning and the validation of the model. Relative errors for stationary measurements between 
a measured variable $y_{\text {meas,stat }}$ and a modelled variable $y_{\text {mod,stat }}$ are calculated as

$$
\begin{aligned}
& \text { Stationary relative error }(i) \\
& =\frac{y_{\text {meas,stat }}(i)-y_{\text {mod,stat }}(i)}{1 / N \sum_{i=1}^{N} y_{\text {meas,stat }}(i)}
\end{aligned}
$$

where $i$ is an operating point. Relative errors for dynamic measurements between a measured variable $y_{\text {meas,dyn }}$ and a modelled variable $y_{\text {mod,dyn }}$ are calculated as

$$
\begin{aligned}
& \text { Dynamic relative error }(j) \\
& =\frac{y_{\text {meas,dyn }}(j)-y_{\text {mod,dyn }}(j)}{1 / N \sum_{i=1}^{N} y_{\text {meas,stat }}(i)}
\end{aligned}
$$

where $j$ is a time sample. In order to make a fair comparison between these relative errors, both the stationary relative error and the dynamic relative error have the same stationary measurement in the denominator and the mean value of this stationary measurement is calculated in order to avoid large relative errors when $y_{\text {measstat }}$ is small.

\section{MANIFOLDS}

The intake and exhaust manifolds are modelled as dynamic systems with two states each, and these are pressure and oxygen mass fraction. The standard isothermal model [1], which is based upon mass conservation, the ideal-gas law, and the fact that the manifold temperature is constant or varies slowly, gives the differential equations for the manifold pressures as

$$
\begin{aligned}
\frac{\mathrm{d}}{\mathrm{d} t} p_{\mathrm{im}} & =\frac{R_{\mathrm{a}} T_{\mathrm{im}}}{V_{\mathrm{im}}}\left(W_{\mathrm{c}}+W_{\mathrm{egr}}-W_{\mathrm{ei}}\right) \\
\frac{\mathrm{d}}{\mathrm{d} t} p_{\mathrm{em}} & =\frac{R_{\mathrm{e}} T_{\mathrm{em}}}{V_{\mathrm{em}}}\left(W_{\mathrm{eo}}-W_{\mathrm{t}}-W_{\mathrm{egr}}\right)
\end{aligned}
$$

There are two sets of thermodynamic properties: air has the ideal-gas constant $R_{\mathrm{a}}$ and the specific heat capacity ratio $\gamma_{\mathrm{a}}$, exhaust gas has the ideal-gas constant $R_{\mathrm{e}}$ and the specific heat capacity ratio $\gamma_{\mathrm{e}}$. The intake manifold temperature $T_{\mathrm{im}}$ is assumed to be constant and equal to the cooling temperature in the intercooler, the exhaust manifold temperature $T_{\text {em }}$ will be described in section 4.2, and $V_{\mathrm{im}}$ and $V_{\mathrm{em}}$ are the manifold volumes. The mass flows $W_{\mathrm{c}}$, $W_{\text {egr }}, W_{\text {ei }}, W_{\text {eo }}$, and $W_{\mathrm{t}}$ will be described in sections 4 to 6 .
The EGR fraction in the intake manifold is calculated as

$$
x_{\text {egr }}=\frac{W_{\text {egr }}}{W_{c}+W_{\text {egr }}}
$$

Note that the EGR gas also contains oxygen which affects the oxygen-to-fuel ratio in the cylinder. This effect is considered by modelling the oxygen concentrations $X_{\text {Oim }}$ and $X_{\text {Oem }}$ in the control volumes. These concentrations are defined in the same way as in reference [4] according to

$$
X_{\text {Oim }}=\frac{m_{\text {Oim }}}{m_{\text {totim }}}, \quad X_{\text {Oem }}=\frac{m_{\text {Oem }}}{m_{\text {totem }}}
$$

where $m_{\text {Oim }}$ and $m_{\text {Oem }}$ are the oxygen masses in the intake manifold and exhaust manifold respectively, and $m_{\text {totim }}$ and $m_{\text {totem }}$ are the total masses in the intake manifold and exhaust manifolds respectively. Differentiating $X_{\mathrm{Oim}}$ and $X_{\mathrm{Oem}}$ and using mass conservation [4] give the differential equations

$$
\begin{aligned}
\frac{\mathrm{d}}{\mathrm{d} t} X_{\text {Oim }}= & \frac{R_{\mathrm{a}} T_{\mathrm{im}}}{p_{\mathrm{im}} V_{\mathrm{im}}}\left(\left(X_{\text {Oem }}-X_{\text {Oim }}\right) W_{\text {egr }}\right. \\
& \left.+\left(X_{\mathrm{Oc}}-X_{\text {Oim }}\right) W_{\mathrm{c}}\right) \\
\frac{\mathrm{d}}{\mathrm{d} t} X_{\text {Oem }}= & \frac{R_{\mathrm{e}} T_{\mathrm{em}}}{p_{\mathrm{em}} V_{\mathrm{em}}}\left(X_{\text {Oe }}-X_{\text {Oem }}\right) W_{\text {eo }}
\end{aligned}
$$

where $X_{\mathrm{Oc}}$ is the constant oxygen concentration passing through the compressor, i.e. air with $X_{\mathrm{Oc}}=23.14$ per cent, and $X_{\mathrm{Oe}}$ is the oxygen concentration in the exhaust gases coming from the engine cylinders, $X_{\mathrm{Oe}}$ will be described in section 4.1.

Another way to consider the oxygen in the EGR gas is to model the burned gas ratio $F_{x}$ in the control volume $x$ which is a frequent choice for states in many papers $[\mathbf{2}, \mathbf{1 8}, \mathbf{2 0}]$. The relation between the oxygen concentration $X_{\mathrm{O} x}$ and the burned gas ratio $F_{x}$ in the control volume $x$ is

$$
X_{\mathrm{O} x}=X_{\mathrm{Oc}}\left(1-F_{x}\right)
$$

Inserting this relation into equation (9), the differential equations in references [2], [18], and [20] are obtained. Consequently, the states $X_{\mathrm{O} x}$ and $F_{x}$ are equivalent.

\subsection{Manifold volumes}

Tuning parameters. The tuning parameters are the intake manifold volumes $V_{\mathrm{im}}$ and the exhaust manifold volume $V_{\mathrm{em}}$.

Tuning method. The tuning parameters $V_{\mathrm{im}}$ and $V_{\mathrm{em}}$ are determined using least-squares optimization for the complete model and using data from 
the dynamic measurements in the data set B and C in Table 1, see section 8.1 for more details.

\section{CYLINDER}

Three submodels describe the behaviour of the cylinder; these are as follows:

(a) a mass flow model that describes the gas and fuel flows that enter and leave the cylinder, the oxygen-to-fuel ratio, and the oxygen concentration out from the cylinder;

(b) a model of the exhaust manifold temperature;

(c) an engine torque model.

\subsection{Cylinder flow}

The total mass flow $W_{\text {ei }}$ from the intake manifold into the cylinders is modelled using the volumetric efficiency $\eta_{\mathrm{vol}}[\mathbf{1}]$ and is given by

$$
W_{\mathrm{ei}}=\frac{\eta_{\mathrm{vol}} p_{\mathrm{im}} n_{\mathrm{e}} V_{\mathrm{d}}}{120 R_{\mathrm{a}} T_{\mathrm{im}}}
$$

where $p_{\mathrm{im}}$ and $T_{\mathrm{im}}$ are the pressure and temperature respectively in the intake manifold, $n_{\mathrm{e}}$ is the engine speed, and $V_{\mathrm{d}}$ is the displaced volume. The volumetric efficiency is in its turn modelled as

$$
\eta_{\mathrm{vol}}=c_{\mathrm{vol} 1} \sqrt{p_{\mathrm{im}}}+c_{\mathrm{vol} 2} \sqrt{n_{\mathrm{e}}}+c_{\mathrm{vol} 3}
$$

The fuel mass flow $W_{\mathrm{f}}$ into the cylinders is controlled by $u_{\delta}$, which gives the injected mass of fuel in milligrams per cycle and cylinder as

$$
W_{\mathrm{f}}=\frac{10^{-6}}{120} u_{\delta} n_{\mathrm{e}} n_{\mathrm{cyl}}
$$

where $n_{\text {cyl }}$ is the number of cylinders. The mass flow $W_{\mathrm{eo}}$ out from the cylinder is given by the mass balance as

$$
W_{\mathrm{eo}}=W_{\mathrm{f}}+W_{\mathrm{ei}}
$$

The oxygen-to-fuel ratio $\lambda_{\mathrm{O}}$ in the cylinder is defined as

$$
\lambda_{\mathrm{O}}=\frac{W_{\mathrm{ei}} X_{\text {Oim }}}{W_{\mathrm{f}}(\mathrm{O} / \mathrm{F})_{\mathrm{s}}}
$$

where $(\mathrm{O} / \mathrm{F})_{\mathrm{s}}$ is the stoichiometric ratio of the oxygen mass to the fuel mass. The oxygen-to-fuel ratio is equivalent to the air-to-fuel ratio which is a common choice of performance variable in the literature [18, 20-22].

During the combustion, the oxygen is burned in the presence of fuel. In diesel engines, $\lambda_{\mathrm{O}}>1$ to avoid smoke. Therefore, it is assumed that $\lambda_{\mathrm{O}}>1$ and the oxygen concentration out from the cylinder can then be calculated as the unburned oxygen fraction

$$
X_{\mathrm{Oe}}=\frac{W_{\mathrm{ei}} X_{\mathrm{Oim}}-W_{\mathrm{f}}(\mathrm{O} / F)_{\mathrm{s}}}{W_{\mathrm{eo}}}
$$

Tuning parameters. The tuning parameters are the volumetric efficiency constants $c_{\mathrm{vol} 1}, c_{\mathrm{vol} 2}, c_{\mathrm{vol} 3}$.

Initialization method. The tuning parameters $c_{\mathrm{vol} 1}, c_{\mathrm{vol} 2}$, and $c_{\mathrm{vol} 3}$ are initialized by solving a linear least-squares problem that minimizes $\left(W_{\mathrm{ei}}-W_{\mathrm{ei}, \mathrm{meas}}\right)^{2}$ with $c_{\mathrm{vol} 1}, c_{\mathrm{vol} 2}$, and $c_{\mathrm{vol} 3}$ as the optimization variables. The variable $W_{\mathrm{ei}}$ is the model in equations (11) and (12) and $W_{\text {ei,meas }}$ is calculated from stationary measurements as $W_{\mathrm{e} i \text {,meas }}=W_{\mathrm{c}} /$ $\left(1-x_{\text {egr }}\right)$. Stationary measurements are used as inputs to the model during the tuning. The result of the initialization is that the cylinder mass flow model has a mean absolute relative error of 0.9 per cent and a maximum absolute relative error of 2.5 per cent. The parameters are then tuned according to the method in section 8.1.

\subsection{Exhaust manifold temperature}

The exhaust manifold temperature model consists of a model for the cylinder-out temperature and a model for the heat losses in the exhaust pipes.

\subsubsection{Cylinder-out temperature}

The cylinder-out temperature $T_{\mathrm{e}}$ is modelled in the same way as in reference [23]. This approach is based upon ideal-gas Seliger cycle (or limited pressure cycle [1] ) calculations that give the cylinderout temperature as

$$
\begin{aligned}
T_{\mathrm{e}}= & \eta_{\mathrm{sc}} \Pi_{\mathrm{e}}^{1-1 / \gamma_{\mathrm{a}}} r_{\mathrm{c}}^{1-\gamma_{\mathrm{a}}} x_{\mathrm{p}}^{1 / \gamma_{\mathrm{a}}-1} \\
& \times\left(q_{\mathrm{in}}\left(\frac{1-x_{\mathrm{cv}}}{c_{p \mathrm{a}}}+\frac{x_{\mathrm{cv}}}{c_{V \mathrm{a}}}\right)+T_{1} r_{\mathrm{c}}^{\gamma_{\mathrm{a}}-1}\right)
\end{aligned}
$$

where $\eta_{\mathrm{sc}}$ is a compensation factor for non-ideal cycles and $x_{\mathrm{cv}}$ the ratio of fuel consumed during constant-volume combustion. The rest of the fuel, i.e. $\left(1-x_{\mathrm{cv}}\right)$ is used during constant-pressure combustion. The model (17) also includes the following six components: the pressure ratio over the cylinder given by 


$$
\Pi_{\mathrm{e}}=\frac{p_{\mathrm{em}}}{p_{\mathrm{im}}}
$$

the pressure ratio between point 3 (after combustion) and point 2 (before combustion) in the Seliger cycle given by

$$
x_{p}=\frac{p_{3}}{p_{2}}=1+\frac{q_{\text {in }} x_{\mathrm{cv}}}{c_{V \mathrm{a}} T_{1} r_{\mathrm{c}}^{\gamma_{\mathrm{a}}-1}}
$$

the specific energy contents of the charge given by

$$
q_{\text {in }}=\frac{W_{\mathrm{f}} q_{\mathrm{H} V}}{W_{\mathrm{ei}}+W_{\mathrm{f}}}\left(1-x_{\mathrm{r}}\right)
$$

the temperature when the inlet valve closes after the intake stroke and mixing given by

$$
T_{1}=x_{\mathrm{r}} T_{\mathrm{e}}+\left(1-x_{\mathrm{r}}\right) T_{\mathrm{im}}
$$

the residual gas fraction given by

$$
x_{\mathrm{r}}=\frac{\Pi_{\mathrm{e}}^{1 / \gamma_{\mathrm{a}}} x_{p}^{-1 / \gamma_{\mathrm{a}}}}{r_{\mathrm{c}} x_{v}}
$$

and the volume ratio between point 3 (after combustion) and point 2 (before combustion) in the Seliger cycle given by

$$
x_{v}=\frac{v_{3}}{v_{2}}=1+\frac{q_{\text {in }}\left(1-x_{\mathrm{cv}}\right)}{c_{p \mathrm{a}}\left[\left(q_{\mathrm{in}} x_{\mathrm{cv}} / c_{V \mathrm{a}}\right)+T_{1} r_{\mathrm{c}}^{\gamma_{\mathrm{a}}-1}\right]}
$$

\subsubsection{Solution to the cylinder-out temperature}

Since the equations above are non-linear and depend on each other, the cylinder-out temperature is calculated numerically using a fixed-point iteration which starts with the initial values $x_{\mathrm{r}, 0}$ and $T_{1,0}$. Then the equations

$$
\begin{aligned}
q_{\mathrm{in}, k+1}= & \frac{W_{\mathrm{f}} q_{\mathrm{H} V}}{W_{\mathrm{ei}}+W_{\mathrm{f}}}\left(1-x_{\mathrm{r}, k}\right) \\
x_{p, k+1}= & 1+\frac{q_{\mathrm{in}, k+1} x_{\mathrm{cv}}}{c_{V \mathrm{a}} T_{1, k} r_{\mathrm{c}}^{\gamma_{\mathrm{a}}-1}} \\
x_{v, k+1}= & 1+\frac{q_{\mathrm{in}, k+1}\left(1-x_{\mathrm{cv}}\right)}{c_{p \mathrm{a}}\left(\left[q_{\mathrm{in}, k+1} x_{\mathrm{cv}} / c_{V \mathrm{a}}\right]+T_{1, k} r_{\mathrm{c}}^{\gamma_{\mathrm{a}}-1}\right)} \\
x_{r, k+1}= & \frac{\Pi_{\mathrm{e}}^{1 / \gamma_{\mathrm{a}}} x_{p, k+1}^{-1 / \gamma_{\mathrm{a}}}}{r_{\mathrm{c}} x_{v, k+1}} \\
T_{\mathrm{e}, k+1}= & \eta_{\mathrm{sc}} \Pi_{\mathrm{e}}^{1-1 / \gamma_{\mathrm{a}}} r_{\mathrm{c}}^{1-\gamma_{\mathrm{a}}} x_{p, k+1}^{1 / \gamma_{\mathrm{a}}-1} \\
& \times\left(q_{\mathrm{in}, \mathrm{k}+1}\left(\frac{1-x_{\mathrm{cv}}}{c_{p \mathrm{a}}}+\frac{x_{\mathrm{cv}}}{c_{V \mathrm{a}}}\right)+T_{1, k} r_{\mathrm{c}}^{\gamma_{\mathrm{a}}-1}\right) \\
T_{1, k+1}= & x_{\mathrm{r}, k+1} T_{\mathrm{e}, k+1}+\left(1-x_{\mathrm{r}, k+1}\right) T_{\mathrm{im}}
\end{aligned}
$$

are applied in each iteration $k$. In each sample during the simulation, the initial values $x_{\mathrm{r}, 0}$ and $T_{1,0}$ are set to the solutions of $x_{\mathrm{r}}$ and $T_{1}$ from the previous sample.

\subsubsection{Approximating the solution to the cylinder-out temperature}

A simulation of the complete engine model during the European transient cycle in Fig. 2 shows that it is sufficient to use one iteration in the iterative process (24). This is shown by comparing the solution from one iteration with one solution that has sufficiently many iterations to give a solution with 0.01 per cent accuracy. The maximum absolute relative error of the solution from one iteration (compared with the solution with 0.01 per cent accuracy) is 0.15 percent. This error is small because the fixed-point iteration (24) has initial values that are close to the solution. Consequently, when using this method in a simulation, it is sufficient to use one iteration in this model since the mean absolute relative error of the exhaust manifold temperature model compared with measurements is 1.7 per cent.

\subsubsection{Heat losses in the exhaust pipes}

The cylinder-out temperature model above does not describe the exhaust manifold temperature completely owing to heat losses in the exhaust pipes between the cylinder and the exhaust manifold. Therefore the next step is to include a submodel for these heat losses.

This temperature drop is modelled in the same way as model 1 presented in reference [24], where the temperature drop is described as a function of mass flow out from the cylinder according to

$$
T_{\mathrm{em}}=T_{\mathrm{amb}}+\left(T_{\mathrm{e}}-T_{\mathrm{amb}}\right) \exp ^{-\frac{h_{\mathrm{tot}} \pi d_{\text {pipe }} l_{\text {pipe }} n_{\text {pipe }}}{W_{\text {eo }} c_{\text {pe }}}}
$$

where $T_{\mathrm{amb}}$ is the ambient temperature, $h_{\mathrm{tot}}$ the total heat transfer coefficient, $d_{\text {pipe }}$ the pipe diameter, $l_{\text {pipe }}$ the pipe length and $n_{\text {pipe }}$ the number of pipes.

Tuning parameters. The tuning parameters are the compensation factor $\eta_{\mathrm{sc}}$ for non-ideal cycles, the ratio $x_{\mathrm{cv}}$ of fuel consumed during constantvolume combustion, and the total heat transfer coefficient $h_{\text {tot }}$.

Initialization method. The tuning parameters $\eta_{\mathrm{sc}}$, $x_{\mathrm{cv}}$, and $h_{\mathrm{tot}}$ are initialized by solving a non-linear least-squares problem that minimizes $\left(T_{\mathrm{em}}-\right.$ $\left.T_{\text {em,meas }}\right)^{2}$ with $\eta_{\mathrm{sc}}, x_{\mathrm{cv}}$, and $h_{\mathrm{tot}}$ as the optimization variables. The variable $T_{\mathrm{em}}$ is the model in equations (24) and (25) with stationary measurements as inputs to the model, and $T_{\mathrm{em}}$,meas is a stationary 
(a)

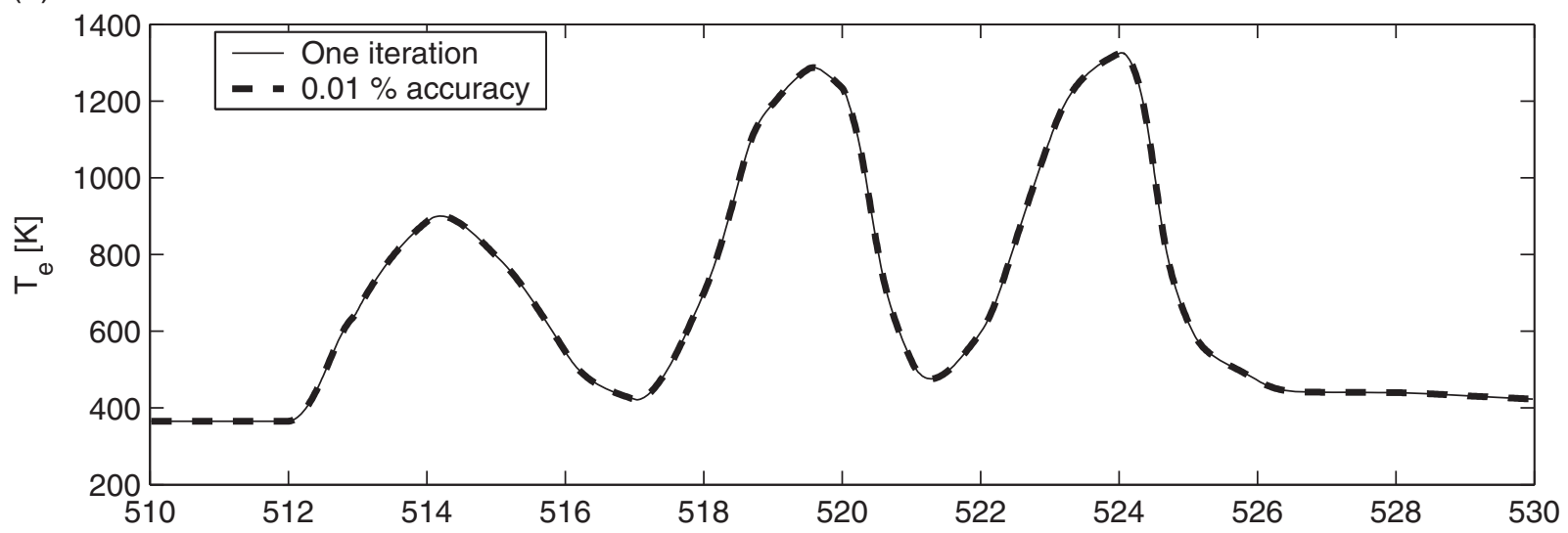

(b)

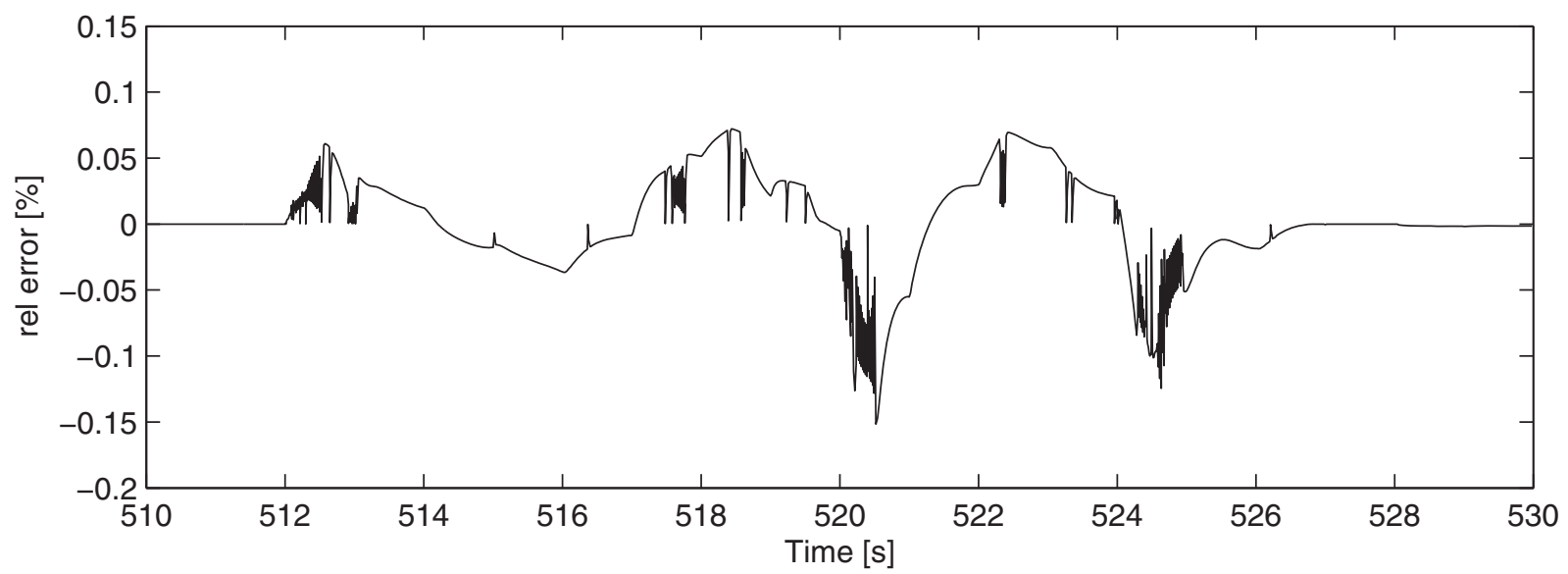

Fig. 2 The cylinder-out temperature $T_{\mathrm{e}}$ calculated by simulating the total engine model during the complete European transient cycle. This figure shows the part of the European transient cycle which consists of the maximum relative error. (a) The fixed-point iteration (24) is used in two ways: by using one iteration and to obtain 0.01 per cent accuracy. (b) Relative errors between the solutions from one iteration and 0.01 per cent accuracy

measurement. The result of the initialization is that the model describes the exhaust manifold temperature well, with a mean absolute relative error of 1.7 per cent and a maximum absolute relative error of 5.4 percent. The parameters are then tuned according to the method in section 8.1.

\subsection{Engine torque}

The torque $M_{\mathrm{e}}$ produced by the engine is modelled using three different engine components, namely the gross indicated torque $M_{\mathrm{ig}}$, the pumping torque $M_{\mathrm{p}}$, and the friction torque $M_{\text {fric }}$ [1] , according to

$$
M_{\mathrm{e}}=M_{\mathrm{ig}}-M_{\mathrm{p}}-M_{\text {fric }}
$$

The pumping torque is modelled using the intake and exhaust manifold pressures, according to

$$
M_{\mathrm{p}}=\frac{V_{\mathrm{d}}}{4 \pi}\left(p_{\mathrm{em}}-p_{\mathrm{im}}\right)
$$

The gross indicated torque is coupled to the energy that comes from the fuel according to

$$
M_{\mathrm{ig}}=\frac{u_{\delta} 10^{-6} n_{\mathrm{cyl}} q_{\mathrm{HV}} \eta_{\mathrm{ig}}}{4 \pi}
$$

Assuming that the engine is always running at optimal injection timing, the gross indicated efficiency $\eta_{\mathrm{ig}}$ is modelled as

$$
\eta_{\text {ig }}=\eta_{\text {igch }}\left(1-\frac{1}{r_{\mathrm{c}}^{\gamma_{\mathrm{cyl}}-1}}\right)
$$

where the parameter $\eta_{\text {igch }}$ is estimated from measurements, $r_{\mathrm{c}}$ is the compression ratio, and $\gamma_{\text {cyl }}$ is the specific heat capacity ratio for the gas in the 
cylinder. The friction torque is assumed to be a quadratic polynomial in engine speed [1] given by

$$
M_{\text {fric }}=\frac{V_{\mathrm{d}}}{4 \pi} 10^{5}\left(\mathrm{c}_{\text {fric } 1} n_{\text {eratio }}^{2}+c_{\text {fric } 2} n_{\text {eratio }}+\mathrm{c}_{\text {fric } 3}\right)
$$

where

$$
n_{\text {eratio }}=\frac{n_{\mathrm{e}}}{1000}
$$

Tuning parameters. The tuning parameters are the combustion chamber efficiency $\eta_{\text {igch }}$ and the coefficients $c_{\text {fric } 1}, c_{\text {fric } 2}$, and $c_{\text {fric } 3}$ in the polynomial function for the friction torque.

Tuning method. The tuning parameters $\eta_{\mathrm{igch}}$, $c_{\text {fric1 } 1}, \quad c_{\text {fric2 }}$, and $c_{\text {fric3 }}$ are determined by solving a linear least-squares problem that minimizes $\left(M_{\mathrm{e}}+M_{\mathrm{p}}-M_{\mathrm{e}, \text { meas }}-M_{\mathrm{p} \text {,meas }}\right)^{2}$ with the tuning parameters as the optimization variables. The model of $M_{\mathrm{e}}+M_{\mathrm{p}}$ is obtained by solving equation (26) for $M_{\mathrm{e}}+M_{\mathrm{p}}$ and $M_{\mathrm{e} \text {,meas }}+M_{\mathrm{p} \text {,meas }}$ is calculated from stationary measurements as $M_{\mathrm{e} \text {,meas }}+M_{\mathrm{p} \text {,meas }}=$ $M_{\mathrm{e}}+V_{\mathrm{d}}\left(p_{\mathrm{em}}-p_{\mathrm{im}}\right) /(4 \pi)$. Station-ary measurements are used as inputs to the model. The result of the tuning is that the engine torque model has small absolute relative errors with a mean absolute relative error of 1.9 percent and a maximum absolute relative error of 7.1 per cent.

\section{EGR VALVE}

The EGR-valve model consists of submodels for the EGR-valve mass flow and the EGR-valve actuator.

\subsection{EGR-valve mass flow}

The mass flow through the EGR valve is modelled as a simplification of a compressible flow restriction with variable area [1] and with the assumption that there is no reverse flow when $p_{\mathrm{em}}<p_{\mathrm{im}}$. The motive for this assumption is to construct a simple model. The model can be extended with reverse flow, but this increases the complexity of the model since a reverse-flow model requires mixing of different temperatures and oxygen fractions in the exhaust manifold and a change in the temperature and the gas constant in the EGR mass flow model. However, $p_{\mathrm{em}}$ is smaller than $p_{\mathrm{im}}$ only in a small operating region with low engine speeds, high engine torque, and half-open to fully open VGT where the engine almost never operates [9]. In addition, reverse flow is also avoided during normal operation by closing the EGR valve at these points.

The mass flow through the restriction is

$$
W_{\mathrm{egr}}=\frac{A_{\mathrm{egr}} p_{\mathrm{em}} \Psi_{\mathrm{egr}}}{\sqrt{T_{\mathrm{em}} R_{\mathrm{e}}}}
$$

where

$$
\Psi_{\mathrm{egr}}=\sqrt{\frac{2 \gamma_{\mathrm{e}}}{\gamma_{\mathrm{e}}-1}\left(\Pi_{\mathrm{egr}}^{2 / \gamma_{\mathrm{e}}}-\Pi_{\mathrm{egr}}^{1+1 / \gamma_{\mathrm{e}}}\right)}
$$

Measurement data show that equation (33) does not give a sufficiently accurate description of the EGR flow. Pressure pulsations in the exhaust manifold or the influence of the EGR cooler could be two different explanations for this phenomenon. In order to maintain the density influence $\left(p_{\mathrm{em}} /\left(\sqrt{T_{\mathrm{em}} R_{\mathrm{e}}}\right)\right)$ in equation (32) and the simplicity of the model, the function $\Psi_{\text {egr }}$ is instead modelled as a parabolic function

$$
\Psi_{\text {egr }}=1-\left(\frac{1-\Pi_{\text {egr }}}{1-\Pi_{\text {egropt }}}-1\right)^{2}
$$

This submodel is compared with calculated points from measurements of Fig. 3(a) where the points are calculated by solving equation (32) for $\Psi_{\text {egr }}$. Note that this comparison is not a validation of the submodel since the calculated points depend on the model tuning.

The pressure ratio $\Pi_{\text {egr }}$ over the valve is limited when the flow is choked, i.e. when sonic conditions are reached in the throat, and when $1<p_{\mathrm{im}} / p_{\mathrm{em}}$, i.e. no back flow can occur, according to

$$
\Pi_{\text {egr }}=\left\{\begin{array}{cl}
\Pi_{\text {egropt }} & \text { if } \frac{p_{\text {im }}}{p_{\text {em }}}<\Pi_{\text {egropt }} \\
\frac{p_{\text {im }}}{p_{\text {em }}} & \text { if } \Pi_{\text {egropt }} \leqslant \frac{p_{\text {im }}}{p_{\text {em }}} \leqslant 1 \\
1 & \text { if } 1<\frac{p_{\text {im }}}{p_{\text {em }}}
\end{array}\right.
$$

For a compressible flow restriction, the standard model for $\Pi_{\text {egropt }}$ is

$$
\Pi_{\text {egropt }}=\left(\frac{2}{\gamma_{\mathrm{e}}+1}\right)^{\gamma_{\mathrm{e}} / \gamma_{\mathrm{e}}-1}
$$

but the accuracy of the EGR flow model is improved by replacing the physical value of $\Pi_{\text {egropt }}$ in equation (36) with a tuning parameter [25].

The effective area

$$
A_{\text {egr }}=A_{\text {egrmax }} f_{\text {egr }}\left(\tilde{u}_{\text {egr }}\right)
$$

is modelled as a polynomial function of the EGR valve position $\tilde{u}_{\text {egr }}$ according to 
(a)

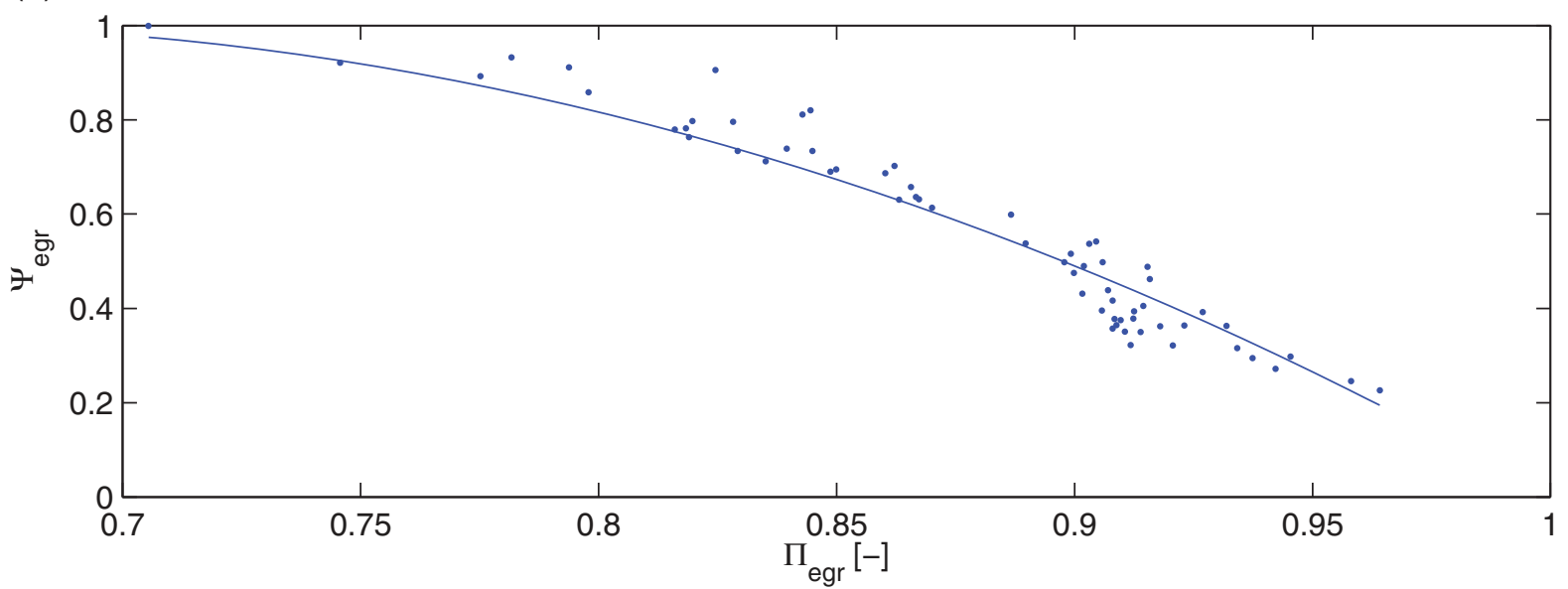

(b)

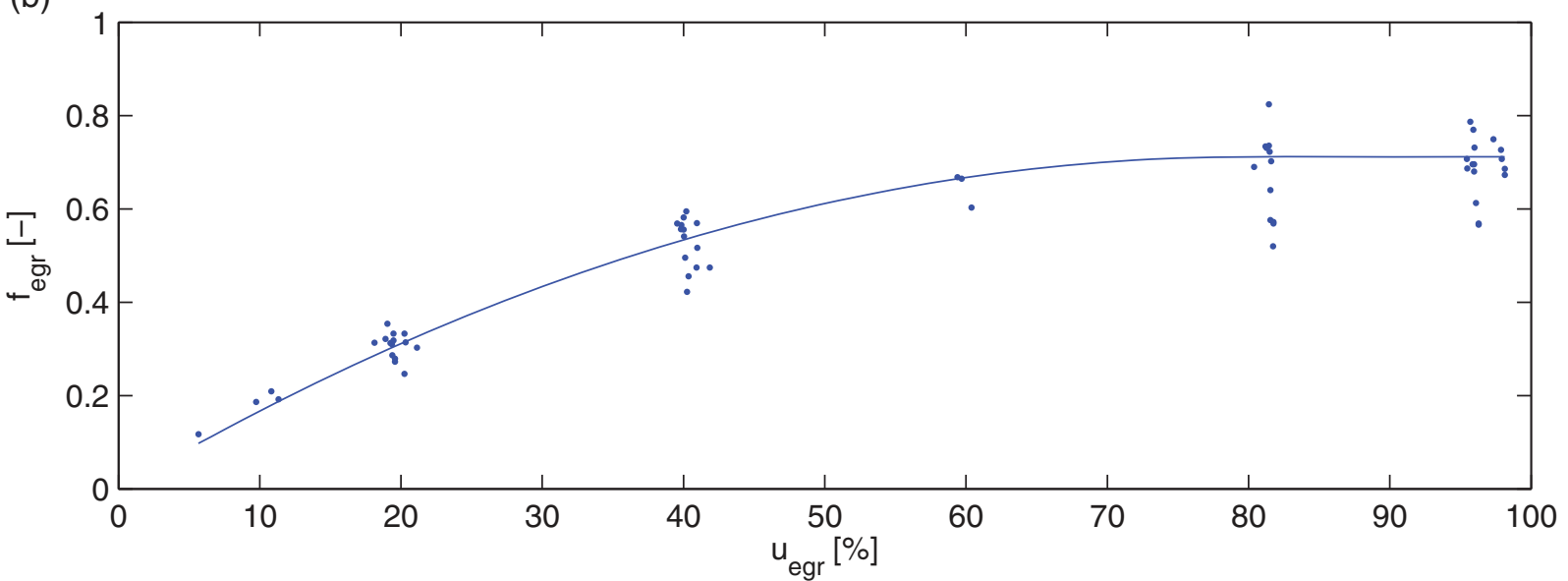

Fig. 3 Comparison of calculated points from measurements and the two submodels given in (a) equation (34) and (b) equation (38) in the EGR flow model at steady state, showing how different variables in the submodels depend on each other

$$
f_{\text {egr }}\left(\tilde{u}_{\text {egr }}\right)= \begin{cases}c_{\text {egr } 1} \tilde{u}_{\text {egr }}^{2}+c_{\text {egr } 2} \tilde{u}_{\text {egr }}+c_{\text {egr } 3} & \text { if } \tilde{u}_{\text {egr }} \leqslant-\frac{c_{\text {egr } 2}}{2 c_{\text {egr } 1}} \\ c_{\text {egr } 3}-\frac{c_{\text {egr } 2}^{2}}{4 c_{\text {egr }}} & \text { if } \tilde{u}_{\text {egr }}>-\frac{c_{\text {egr } 2}}{2 c_{\text {egr } 1}}\end{cases}
$$

where $\tilde{u}_{\text {egr }}$ describes the EGR actuator dynamics, see section 5.2. The EGR valve is open when $\tilde{u}_{\text {egr }}=$ 100 per cent and closed when $\tilde{u}_{\text {egr }}=0$ per cent. The submodel (38) is compared with calculated points from measurements in Fig. 3(b) where the points are calculated by solving equation (32) for $f_{\text {egr }}$. Note that this comparison is not a validation of the submodel since the calculated points depend on the model tuning.

Tuning parameters. The tuning parameters are the optimal value $\Pi_{\text {egropt }}$ of $\Pi_{\text {egr }}$ for the maximum value of the function $\Psi_{\text {egr }}$ in equation (34) and the coefficients $c_{\text {egr1 }}, c_{\text {egr2 }}$, and $c_{\text {egr3 }}$ in the polynomial function for the effective area,
Initialization method. The tuning parameters above are initialized by solving a separable nonlinear least-squares problem; see reference [26] for details of the solution method. The non-linear part of this problem minimizes $\left(W_{\text {egr }}-W_{\text {egr,meas }}\right)^{2}$ with $\Pi_{\text {egropt }}$ as the optimization variable. In each iteration in the non-linear least-squares solver, the values for $c_{\mathrm{egr} 1}, c_{\mathrm{egr} 2}$, and $c_{\mathrm{egr} 3}$ are set to be the solution of a linear least-squares problem that minimizes $\left(W_{\text {egr }}-W_{\text {egr,meas }}\right)^{2}$ for the current value of $\Pi_{\text {egropt. }}$ The variable $W_{\text {egr }}$ is described by the model (32) and $W_{\text {egr,meas }}$ is calculated from measurements as $W_{\text {egr,meas }}=W_{\mathrm{c}} x_{\text {egr }} /\left(1-x_{\text {egr }}\right)$. Stationary measurements are used as inputs to the model. The result of the initialization is that the absolute relative errors are larger than 15 percent at some points. However, the model describes the EGR mass flow well at the other points; the mean absolute relative error is 6.1 per cent and the maximum absolute relative error is 22.2 percent. The 


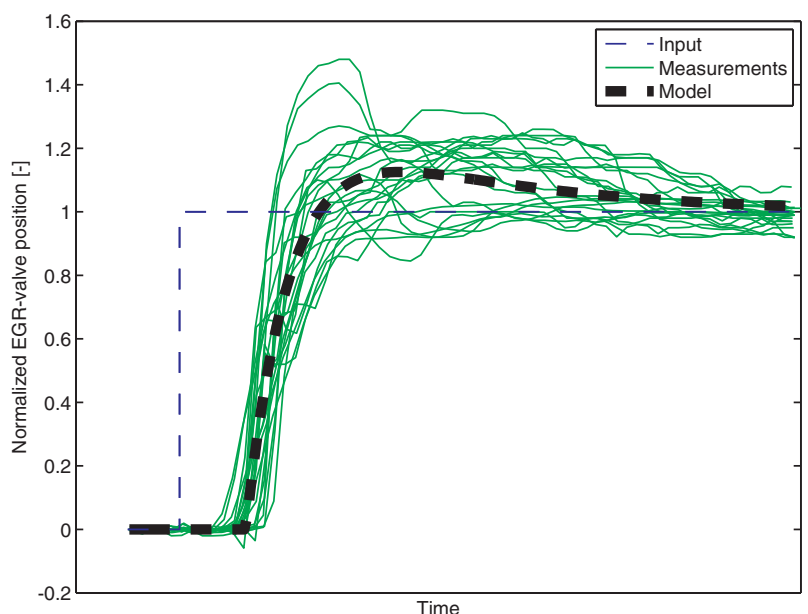

Fig. 4 Comparison between the EGR actuator dynamic simulation and dynamic tuning data during steps in the EGR-valve position

parameters are then tuned according to the method in section 8.1.

\subsection{EGR-valve actuator}

The EGR-valve actuator dynamics are modelled as a second-order system with an overshoot and a time delay (Fig. 4). This model consist of a subtraction between the two first-order systems with different gains and time constants according to

$$
\begin{aligned}
& \tilde{u}_{\text {egr }}=K_{\text {egr }} \tilde{u}_{\text {egr } 1}-\left(K_{\text {egr }}-1\right) \tilde{u}_{\text {egr } 2} \\
& \frac{\mathrm{d}}{\mathrm{d} t} \tilde{u}_{\text {egr } 1}=\frac{1}{\tau_{\text {egr } 1}}\left[u_{\text {egr }}\left(t-\tau_{\text {degr }}\right)-\tilde{u}_{\text {egr } 1}\right] \\
& \frac{\mathrm{d}}{\mathrm{d} t} \tilde{u}_{\text {egr } 2}=\frac{1}{\tau_{\text {egr } 2}}\left[u_{\text {egr }}\left(t-\tau_{\text {degr }}\right)-\tilde{u}_{\text {egr } 2}\right]
\end{aligned}
$$

Tuning parameters. The tuning parameters are the time constants $\tau_{\text {egr } 1}$ and $\tau_{\text {egr } 2}$ for the two different first-order systems, the time delay $\tau_{\text {degr }}$, and a parameter $K_{\text {egr }}$ that affects the size of the overshoot.

Tuning method. The tuning parameters above are determined by solving a non-linear least-squares problem that minimizes $\left(\tilde{u}_{\text {egr }}-\tilde{u}_{\text {egr }}\right.$,meas ${ }^{2}$ with $\tau_{\text {egr } 1}$, $\tau_{\text {egr2 }}, \tau_{\text {degr }}$, and $K_{\text {egr }}$ as the optimization variables. The variable $\tilde{u}_{\text {egr }}$ is the model in equations (39) to (41) and $\tilde{u}_{\text {egr,meas }}$ are dynamic responses in the dynamic data set $\mathrm{J}$ in Table 1 . These data consist of 18 steps in the EGR valve position with a step size of 10 percent, going from 0 percent up to 90 per cent and then back again to 0 percent with a step size of 10 percent. The measurements also consist of one step with a step size of 30 per cent, one step with a step size of 75 percent, three steps with a step size of 80 per cent, and one step with a step size of 90 per cent. These 24 steps are normalized and shifted in time in order to achieve the same starting point. These measurements are then compared with the unit step response for the linear system (39) to (41) in Fig. 4. This shows that the measurements have both large overshoots and no overshoots in some steps. There are no strong dependences between these variations and the position of the EGR valve. Further, the errors between the model and the measurements have very small effects on the errors in the outputs from the complete engine model. This has been investigated by removing the actuator model from the complete model and using measurements of the real actuator position as the input to the complete model. Consequently, the proposed EGR actuator model is a sufficiently close approximation that describes the actuator well on average.

\section{TURBOCHARGER}

The turbocharger consists of a turbo inertia model, a turbine model, a VGT actuator model, and a compressor model.

\subsection{Turbo inertia}

For the turbo speed $\omega_{\mathrm{t}}$, Newton's second law gives

$$
\frac{\mathrm{d}}{\mathrm{dt}} \omega_{\mathrm{t}}=\frac{P_{\mathrm{t}} \eta_{\mathrm{m}}-P_{\mathrm{c}}}{J_{\mathrm{t}} \omega_{\mathrm{t}}}
$$

where $J_{\mathrm{t}}$ is the inertia, $P_{\mathrm{t}}$ is the power delivered by the turbine, $P_{\mathrm{c}}$ is the power required to drive the compressor, and $\eta_{\mathrm{m}}$ is the mechanical efficiency in the turbocharger.

Tuning parameter. The tuning parameter is the turbo inertia $J_{\mathrm{t}}$.

Tuning method. The tuning parameter $J_{\mathrm{t}}$ is determined using least-squares optimization for the complete model and using data from the dynamic measurements in the data sets B and C in Table 1, see section 8.1 for more details.

\subsection{Turbine}

The turbine model consists of submodels for the total turbine efficiency and the turbine mass flow, which also includes the VGT actuator as a submodel.

\subsubsection{Turbine efficiency}

One way to model the power $P_{\mathrm{t}}$ is to use the turbine efficiency $\eta_{\mathrm{t}}$, which is defined as $[\mathbf{1}]$ 


$$
\eta_{\mathrm{t}}=\frac{P_{\mathrm{t}}}{P_{\mathrm{t}, \mathrm{s}}}=\frac{T_{\mathrm{em}}-T_{\mathrm{t}}}{T_{\mathrm{em}}\left(1-\Pi_{\mathrm{t}}^{1-1 / \gamma_{\mathrm{e}}}\right)}
$$

where $T_{\mathrm{t}}$ is the temperature after the turbine, $\Pi_{\mathrm{t}}$ is the pressure ratio given by

$$
\Pi_{\mathrm{t}}=\frac{p_{\mathrm{es}}}{p_{\mathrm{em}}}
$$

and $P_{\mathrm{t}, \mathrm{s}}$ is the power from the isentropic process

$$
P_{\mathrm{t}, \mathrm{s}}=W_{\mathrm{t}} c_{p \mathrm{e}} T_{\mathrm{em}}\left(1-\Pi_{\mathrm{t}}^{1-1 / \gamma_{\mathrm{e}}}\right)
$$

where $W_{\mathrm{t}}$ is the turbine mass flow. In equation (44), $p_{\mathrm{es}}>p_{\mathrm{amb}}$ if there is a restriction (e.g. an aftertreatment system) after the turbine. However, for this engine there is no restriction after the turbine and therefore $p_{\mathrm{es}}=p_{\mathrm{amb}}$.

In equation (43), it is assumed that there are no heat losses in the turbine, i.e. it is assumed that there are no temperature drops between the temperatures $T_{\mathrm{em}}$ and $T_{\mathrm{t}}$ that are due to heat losses. This assumption leads to errors in $\eta_{\mathrm{t}}$ if equation (43) is used to calculate $\eta_{\mathrm{t}}$ from measurements. One way to improve this model is to model these temperature drops, but it is difficult to tune these models since no measurements of these temperature drops exist. Another way to improve the model, that is frequently used in the literature [27], is to use another efficiency that is approximately equal to $\eta_{\mathrm{t}}$. This approximation utilizes the fact that

$$
P_{\mathrm{t}} \eta_{\mathrm{m}}=P_{\mathrm{c}}
$$

at steady state according to equation (42). Consequently, $P_{\mathrm{t}} \approx P_{\mathrm{c}}$ at steady state. Using this approximation in equation (43), another efficiency $\eta_{\mathrm{tm}}$ is obtained as

$$
\eta_{\mathrm{tm}}=\frac{P_{\mathrm{c}}}{P_{\mathrm{t}, \mathrm{s}}}=\frac{W_{\mathrm{c}} c_{p \mathrm{a}}\left(T_{\mathrm{c}}-T_{\mathrm{amb}}\right)}{W_{\mathrm{t}} c_{p \mathrm{e}} T_{\mathrm{em}}\left(1-\Pi_{\mathrm{t}}^{1-1 / \gamma_{\mathrm{e}}}\right)}
$$

where $T_{\mathrm{c}}$ is the temperature after the compressor and $W_{\mathrm{c}}$ is the compressor mass flow. The temperature $T_{\mathrm{em}}$ in equation (47) introduces fewer errors than the temperature difference $T_{\mathrm{em}}-T_{\mathrm{t}}$ in equation (43) does because the absolute value of $T_{\mathrm{em}}$ is larger than the absolute value of $T_{\mathrm{em}}-T_{\mathrm{t}}$. Consequently, equation (47) introduces fewer errors than equation (43) does since equation (47) does not consist of $T_{\mathrm{em}}-T_{\mathrm{t}}$. The temperatures $T_{\mathrm{c}}$ and $T_{\mathrm{amb}}$ are low and they introduce fewer errors than $T_{\mathrm{em}}$ and $T_{\mathrm{t}}$ since the heat losses in the compressor are comparatively small. Another advantage of using equation (47) is that the individual variables $P_{\mathrm{t}}$ and $\eta_{\mathrm{m}}$ in equation (42) do not have to be modelled. Instead, the product $P_{\mathrm{t}} \eta_{\mathrm{m}}$ is modelled using equations (46) and (47) according to

$$
P_{\mathrm{t}} \eta_{\mathrm{m}}=\eta_{\mathrm{tm}} P_{\mathrm{t}, \mathrm{s}}=\eta_{\mathrm{tm}} W_{\mathrm{t}} c_{p \mathrm{e}} T_{\mathrm{em}}\left(1-\Pi_{\mathrm{t}}^{1-1 / \gamma_{\mathrm{e}}}\right)
$$

Measurements show that $\eta_{\mathrm{tm}}$ depends on the blade speed ratio (BSR) as a parabolic function [28], according to

$$
\eta_{\mathrm{tm}}=\eta_{\mathrm{tm}, \max }-c_{\mathrm{m}}\left(\mathrm{BSR}-\mathrm{BSR}_{\mathrm{opt}}\right)^{2}
$$

The BSR is the ratio of the turbine blade tip speed to the speed which a gas reaches when expanded isentropically at the given pressure ratio $\Pi_{\mathrm{t}}$; the BSR is given by

$$
\mathrm{BSR}=\frac{R_{\mathrm{t}} \omega_{\mathrm{t}}}{\sqrt{2 c_{p \mathrm{e}} T_{\mathrm{em}}\left(1-\Pi_{\mathrm{t}}^{1-1 / \gamma_{\mathrm{e}}}\right)}}
$$

where $R_{\mathrm{t}}$ is the turbine blade radius. The parameter $c_{\mathrm{m}}$ in the parabolic function varies owing to mechanical losses, and $c_{\mathrm{m}}$ is therefore modelled as a function of the turbo speed according to

$$
c_{\mathrm{m}}=c_{\mathrm{m} 1}\left[\max \left(0, \omega_{\mathrm{t}}-c_{\mathrm{m} 2}\right)\right]^{c_{\mathrm{m} 3}}
$$

Tuning parameters. The tuning parameters are the maximum turbine efficiency $\eta_{\mathrm{tm} \text {,max }}$, the optimum BSR value $\mathrm{BSR}_{\text {opt }}$ for the maximum turbine efficiency, and the parameters $c_{\mathrm{m} 1}, c_{\mathrm{m} 2}$, and $c_{\mathrm{m} 3}$ in the model for $c_{\mathrm{m}}$.

Initialization method. The tuning parameters above are initialized by solving a separable nonlinear least-squares problem, see reference [26] for details of the solution method. The non-linear part of this problem minimizes $\left(\eta_{\mathrm{tm}}-\eta_{\mathrm{tm} \text {,meas }}\right)^{2}$ with $\mathrm{BSR}_{\mathrm{opt}}, c_{\mathrm{m} 2}$, and $c_{\mathrm{m} 3}$ as the optimization variables. In each iteration in the non-linear least-squares solver, the values for $\eta_{\mathrm{tm}, \max }$ and $c_{\mathrm{m} 1}$ are set to be the solution of a linear least-squares problem that minimizes $\left(\eta_{\mathrm{tm}}-\eta_{\mathrm{tm} \text {,meas }}\right)^{2}$ for the current values of $\mathrm{BSR}_{\mathrm{opt}}, c_{\mathrm{m} 2}$, and $c_{\mathrm{m} 3}$. The efficiency $\eta_{\mathrm{tm}}$ is described by the model (49), and $\eta_{\mathrm{tm} \text {,meas }}$ is calculated from measurements using equation (47). Stationary measurements are used as inputs to the model. The result of the initialization is that the model describes the total turbine efficiency well with a mean absolute relative error of 4.2 percent and a maximum absolute relative error of 13.2 percent. 
(a)

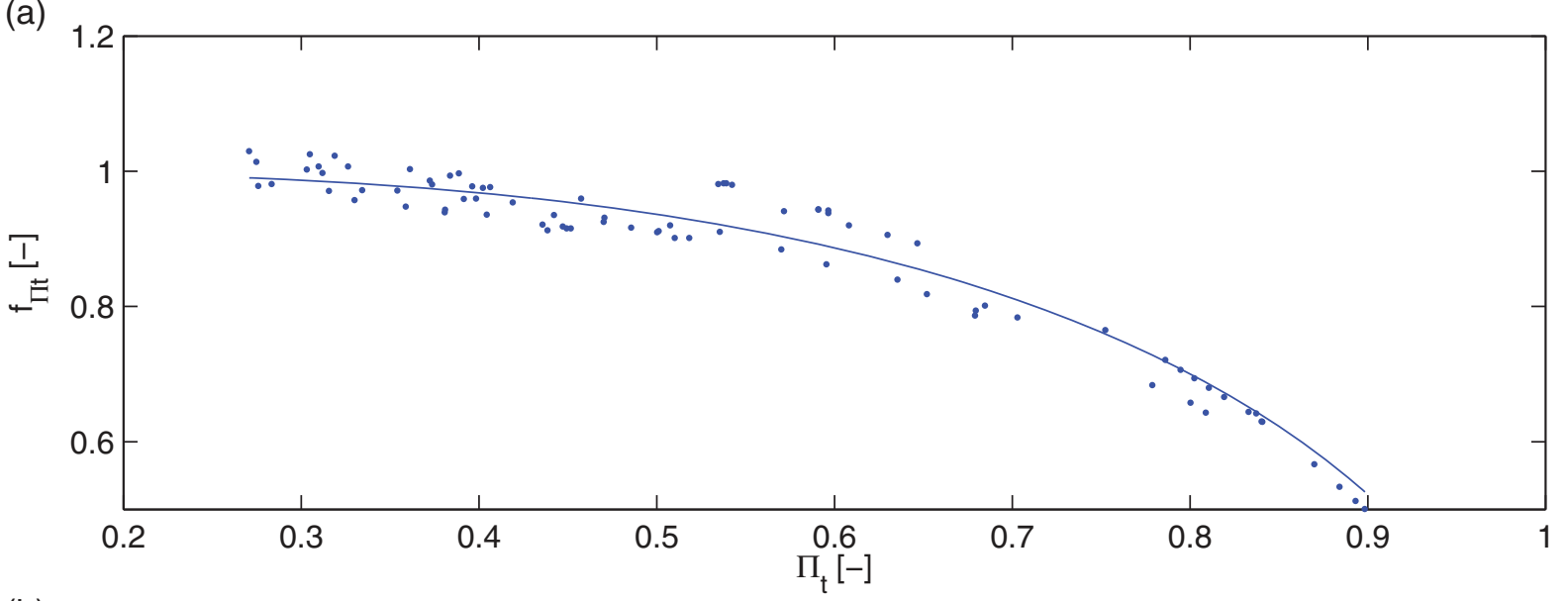

(b)

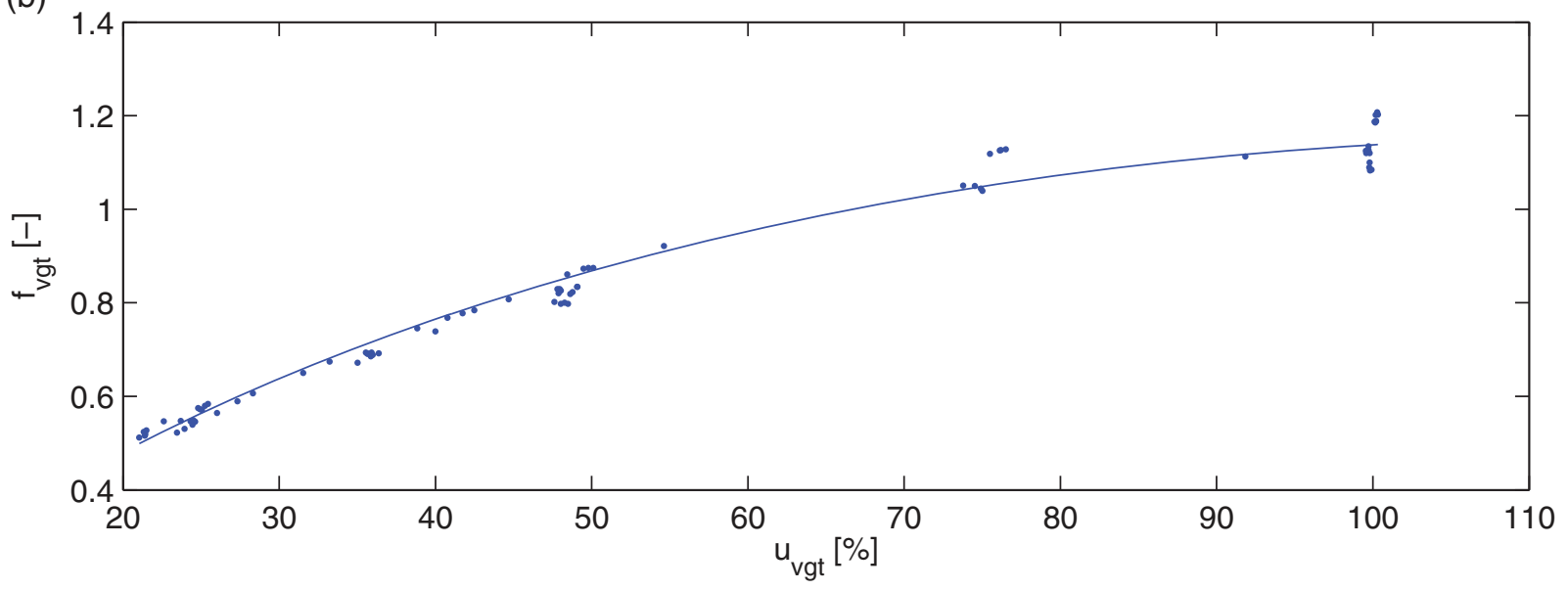

Fig. 5 Comparison of calculated points from measurements and the two submodels given in (a) equation (53) and (b) equation (56) in the turbine mass flow model at steady state showing how different variables in the submodels depend on each other

The parameters are then tuned according to the method in section 8.1.

\subsubsection{Turbine mass flow}

The turbine mass flow $W_{\mathrm{t}}$ is modelled using the corrected mass flow in order to consider density variations in the mass flow $[\mathbf{1}, \mathbf{2 8}]$ according to

$$
\frac{W_{\mathrm{t}} \sqrt{T_{\mathrm{em}} R_{\mathrm{e}}}}{p_{\mathrm{em}}}=A_{\mathrm{vgtmax}} f_{\Pi t}\left(\Pi_{\mathrm{t}}\right) f_{\mathrm{vgt}}\left(\tilde{u}_{\mathrm{vgt}}\right)
$$

where $A_{\text {vgtmax }}$ is the maximum area in the turbine that the gas flows through. Measurements show that the corrected mass flow depends on the pressure ratio $\Pi_{\mathrm{t}}$ and the VGT actuator signal $\tilde{u}_{\mathrm{vgt}}$. As the pressure ratio decreases, the corrected mass flow increases until the gas reaches the sonic condition and the flow is choked. This behaviour can be described by a choking function

$$
f_{\Pi \Pi \mathrm{t}}\left(\Pi_{\mathrm{t}}\right)=\sqrt{1-\Pi_{\mathrm{t}}^{K_{\mathrm{t}}}}
$$

which is not based on the physics of the turbine, but it gives good agreement with measurements using few parameters [29]. The submodel (53) is compared with calculated points from measurements in Fig. 5(a) where the points are calculated by solving equation (52) for $f_{I I t}$. Note that this comparison is not a validation of the submodel since the calculated points depend on the model tuning.

When the VGT control signal $u_{\mathrm{vgt}}$ increases, the effective area increases and hence also the flow increases. Because of the geometry of the turbine, the change in the effective area is large when the VGT control signal is small. This behaviour can be described by a part of an ellipse

$$
\left[\frac{f_{\mathrm{vgt}}\left(\tilde{u}_{\mathrm{vgt}}\right)-c_{\mathrm{f} 2}}{c_{\mathrm{f} 1}}\right]^{2}+\left[\frac{\tilde{u}_{\mathrm{vgt}}-c_{\mathrm{vgt} 2}}{c_{\mathrm{vgt} 1}}\right]^{2}=1
$$




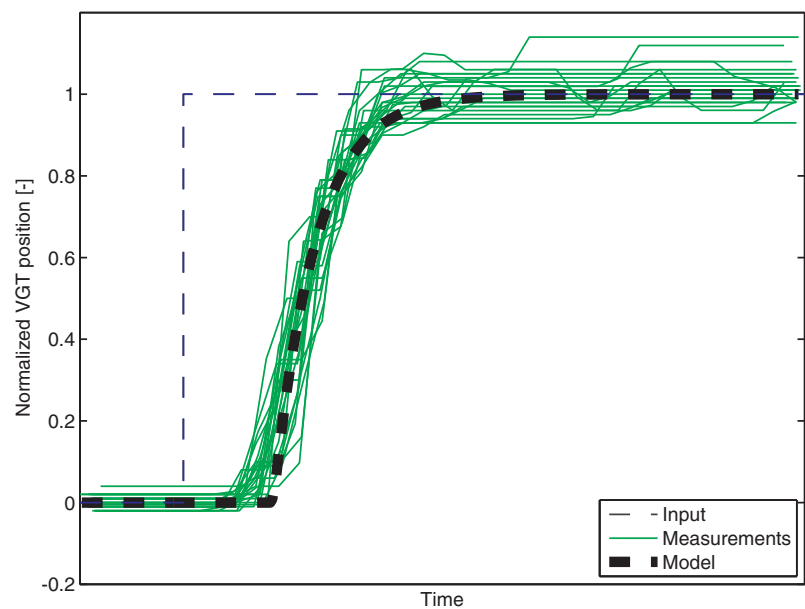

Fig. 6 Comparison between VGT actuator dynamic simulation and dynamic tuning data during steps in the VGT position

where $f_{\text {vgt }}$ is the effective area ratio function and $\tilde{u}_{\text {vgt }}$ describes the VGT actuator dynamics.

The flow can now be modelled by solving equation (52) for $W_{\mathrm{t}}$ giving

$$
W_{\mathrm{t}}=\frac{A_{\mathrm{vgtmax}} p_{\mathrm{em}} f_{\Pi I \mathrm{t}}\left(\Pi_{\mathrm{t}}\right) f_{\mathrm{vgt}}\left(\tilde{u}_{\mathrm{vgt}}\right)}{\sqrt{T_{\mathrm{em}} R_{\mathrm{e}}}}
$$

and solving equation (54) for $f_{\mathrm{vgt}}$, giving

$$
f_{\mathrm{vgt}}\left(\tilde{u}_{\mathrm{vgt}}\right)=c_{\mathrm{f} 2}+c_{\mathrm{f} 1} \sqrt{\max \left(0,1-\left(\frac{\tilde{u}_{\mathrm{vgt}}-c_{\mathrm{vgt} 2}}{c_{\mathrm{vgt}}}\right)^{2}\right)}
$$

The submodel (56) is compared with calculated points from measurements in Fig. 5(b) where the points are calculated by solving equation (52) for $f_{\text {vgt }}$. Note that this comparison is not a validation of the submodel since the calculated points depend on the model tuning.

Tuning parameters. The tuning parameters are the exponent $K_{\mathrm{t}}$ in the choking function for the turbine flow and the parameters $c_{\mathrm{f} 1}, c_{\mathrm{f} 2}, c_{\mathrm{vgf} 1}$, and $c_{\mathrm{vgf} 2}$ in the ellipse for the effective area ratio function.

Initialization method. The tuning parameters above are initialized by solving a non-linear leastsquares problem that minimizes $\left(W_{\mathrm{t}}-W_{\mathrm{t} \text {,meas }}\right)^{2}$ with the tuning parameters as the optimization variables. The flow $W_{\mathrm{t}}$ is described by the model (55), (56), and (53), and $W_{\mathrm{t}, \text { meas }}$ is calculated from measurements as $W_{\mathrm{t}, \text { meas }}=W_{\mathrm{c}}+W_{\mathrm{f}}$, where $W_{\mathrm{f}}$ is calculated using equation (13). Stationary measurements are used as inputs to the model. The result of the initialization is that the turbine mass flow model has small absolute relative errors with a mean absolute relative error of 2.8 percent and a maximum absolute relative error of 7.6 per cent. The parameters are then tuned according to the method in section 8.1.

\subsubsection{VGT actuator}

The VGT actuator dynamics are modelled as a firstorder system with a time delay according to

$$
\frac{\mathrm{d}}{\mathrm{d} t} \tilde{u}_{\mathrm{vgt}}=\frac{1}{\tau_{\mathrm{vgt}}}\left(u_{\mathrm{vgt}}\left(t-\tau_{\mathrm{dvgt}}\right)-\tilde{u}_{\mathrm{vgt}}\right)
$$

Tuning parameters. The tuning parameters are the time constant $\tau_{\text {vgt }}$ and the time delay $\tau_{\text {dvgt }}$.

Tuning method. The tuning parameters above are determined by solving a non-linear least-squares problem that minimizes $\left(\tilde{u}_{\mathrm{vgt}}-\tilde{u}_{\mathrm{vgt}, \mathrm{meas}}\right)^{2}$ with $\tau_{\mathrm{vgt}}$ and $\tau_{\mathrm{dvgt}}$ as the optimization variables. The variable $\tilde{u}_{\mathrm{vgt}}$ is the model in equation (57) and $\tilde{u}_{\mathrm{vgt} \text {,meas }}$ are dynamic responses in the dynamic data set $\mathrm{J}$ in Table 1. These data consist of 18 steps in the VGT position with a step size of 10 per cent, going from 100 percent down to 10 percent and then back again to 100 per cent with a step size of 10 per cent. The measurements also consist of five steps with a step size of 5 per cent and one step with a step size of 20 per cent. These 24 steps are then normalized and shifted in time in order to achieve the same starting point. These measurements are then compared with the unit step response for the linear system (57) in Fig. 6 which shows that the model describes the actuator well.

\subsection{Compressor}

The compressor model consists of submodels for the compressor efficiency and the compressor mass flow.

\subsubsection{Compressor efficiency}

The compressor power $P_{\mathrm{c}}$ is modelled using the compressor efficiency $\eta_{\mathrm{c}}$, which is defined as [1]

$$
\eta_{\mathrm{c}}=\frac{P_{\mathrm{c}, \mathrm{s}}}{P_{\mathrm{c}}}=\frac{T_{\mathrm{amb}}\left(\Pi_{\mathrm{c}}^{1-1 / \gamma_{\mathrm{a}}}-1\right)}{T_{\mathrm{c}}-T_{\mathrm{amb}}}
$$

where $T_{\mathrm{c}}$ is the temperature after the compressor, $\Pi_{\mathrm{c}}$ is the pressure ratio given by

$$
\Pi_{\mathrm{c}}=\frac{p_{\mathrm{im}}}{p_{\mathrm{amb}}}
$$

and $P_{\mathrm{c}, \mathrm{s}}$ is the power from the isentropic process given by 


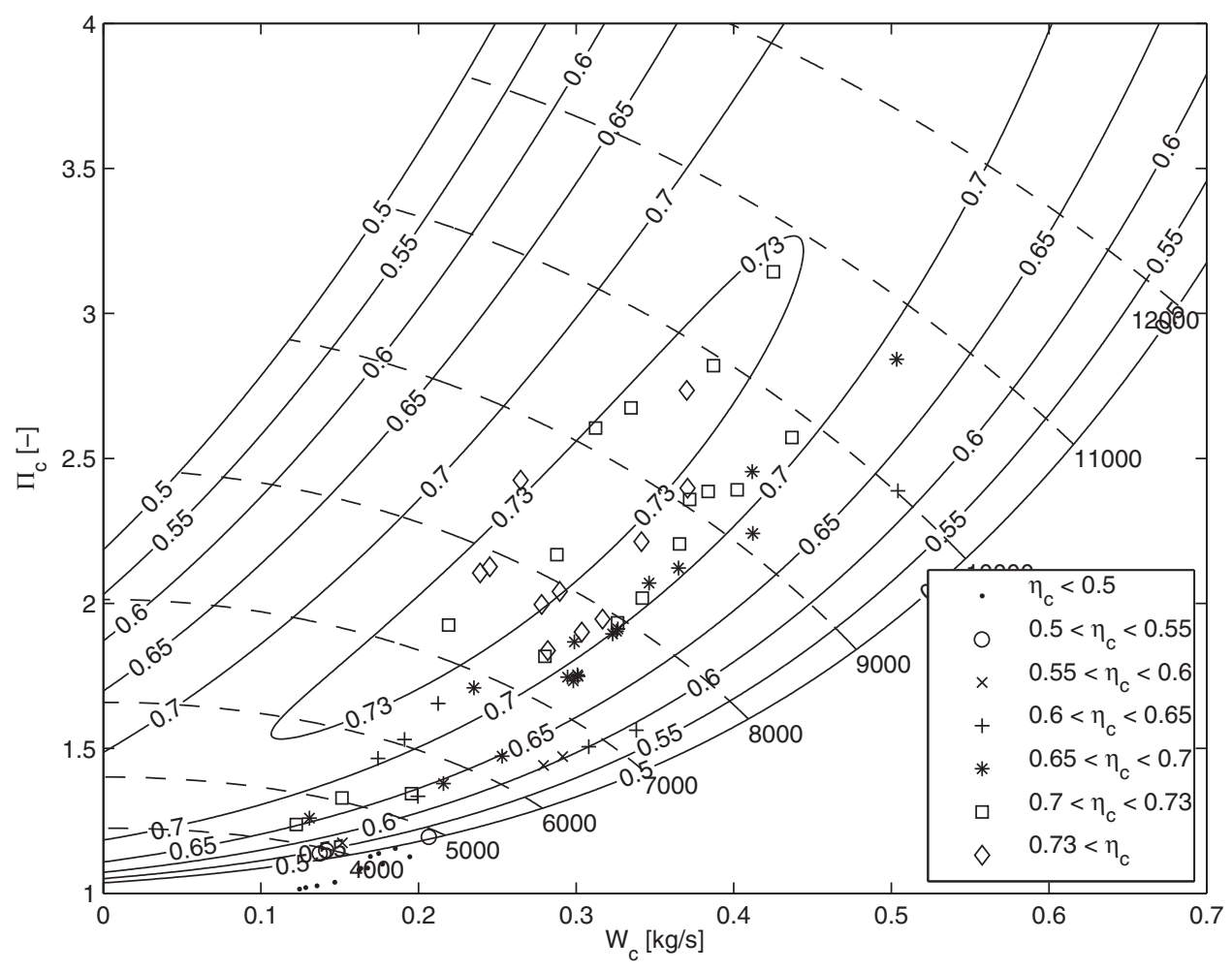

Fig. 7 Compressor map with modelled efficiency lines (solid curves), modelled turbo speed lines (dashed curves with the turbo speed in rads per second), and calculated efficiency from measurements using equation (58). The calculated points are divided into different groups

$$
P_{\mathrm{c}, \mathrm{s}}=W_{\mathrm{c}} c_{p \mathrm{a}} T_{\mathrm{amb}}\left(\Pi_{\mathrm{c}}^{1-1 / \gamma_{\mathrm{a}}}-1\right)
$$

where $W_{\mathrm{c}}$ is the compressor mass flow. The power $P_{\mathrm{c}}$ is modelled by solving equation (58) for $P_{\mathrm{c}}$ and using equation (60) according to

$$
P_{\mathrm{c}}=\frac{P_{\mathrm{c}, \mathrm{s}}}{\eta_{\mathrm{c}}}=\frac{W_{\mathrm{c}} c_{\mathrm{pa}} T_{\mathrm{amb}}}{\eta_{\mathrm{c}}}\left(\Pi_{\mathrm{c}}^{1-1 / \gamma_{\mathrm{a}}}-1\right)
$$

The efficiency is modelled using ellipses similar to the method in reference [30], but with a nonlinear transformation on the axis for the pressure ratio similar to the method in reference [25]. The inputs to the efficiency model are $\Pi_{\mathrm{c}}$ and $W_{\mathrm{c}}$ (see Fig. 7). The flow $W_{\mathrm{c}}$ is not scaled by the inlet temperature and the inlet pressure, in the current implementation, since these two variables are constant. However, this model can easily be extended with a corrected mass flow in order to consider variations in the environmental conditions.

The ellipses can be described as

$$
\eta_{\mathrm{c}}=\eta_{\mathrm{cmax}}-\boldsymbol{\chi}^{\mathrm{T}} \boldsymbol{Q}_{\mathrm{c}} \boldsymbol{\chi}
$$

$\chi$ is a vector which contains the inputs according to

$$
\chi=\left[\begin{array}{l}
W_{\mathrm{c}}-W_{\mathrm{copt}} \\
\pi_{\mathrm{c}}-\pi_{\mathrm{copt}}
\end{array}\right]
$$

where the non-linear transformation for $\Pi_{\mathrm{c}}$ is

$$
\pi_{\mathrm{c}}=\left(\Pi_{\mathrm{c}}-1\right)^{c_{\pi}}
$$

and the symmetric and positive semidefinite matrix $\boldsymbol{Q}_{\text {c }}$ consists of three parameters according to

$$
\boldsymbol{Q}_{\mathrm{c}}=\left[\begin{array}{ll}
a_{1} & a_{3} \\
a_{3} & a_{2}
\end{array}\right]
$$

Tuning model parameters. The tuning model parameters are the maximum compressor efficiency $\eta_{\text {cmax }}$, the optimum value $W_{\text {copt }}$ of $W_{\text {c }}$ and the optimum value $\pi_{\text {copt }}$ of $\pi_{\mathrm{c}}$ for the maximum compressor efficiency, the exponent $c_{\pi}$ in the scale function (64), and the parameters $a_{1}, a_{2}, a_{3}$ in the matrix $\boldsymbol{Q}_{\mathrm{c}}$.

Initialization method. The tuning parameters above are initialized by solving a separable nonlinear least-squares problem; see reference [26] for details of the solution method. The non-linear part of this problem minimizes $\left(\eta_{\mathrm{c}}-\eta_{\mathrm{c}} \text {,meas }\right)^{2}$ with $W_{\text {copt }}$, $\pi_{\text {copt }}$, and $c_{\pi}$ as the optimization variables. In each 
iteration in the non-linear least-squares solver, the values for $\eta_{\mathrm{cmax}}, a_{1}, a_{2}$, and $a_{3}$ are set to be the solution of a linear least-squares problem that minimizes $\left(\eta_{\mathrm{c}}-\eta_{\mathrm{c} \text {, meas }}\right)^{2}$ for the current values of $W_{\text {copt }}, \pi_{\text {copt }}$, and $c_{\pi}$. The efficiency $\eta_{\mathrm{c}}$ is described by the models (62) to (65) and $\eta_{c, \text { meas }}$ is calculated from measurements using equation (58). Stationary measurements are used as inputs to the model. The result of the initialization is that the compressor efficiency model has small absolute relative errors with a mean absolute relative error of 3.3 percent and a maximum absolute relative error of 14.1 percent. The parameters are then tuned according to the method in section 8.1, which guarantees that the matrix $\boldsymbol{Q}_{\mathrm{c}}$ becomes positive semidefinite using the expression in the compressor efficiency model during the tuning given by

$$
a_{3}:=\min \left[\max \left(a_{3},-\sqrt{a_{1} a_{2}}\right), \sqrt{a_{1} a_{2}}\right]
$$

\subsubsection{Compressor mass flow}

The mass flow $W_{\mathrm{c}}$ through the compressor is modelled using two dimensionless variables. The first variable is the energy transfer coefficient [31]

$$
\Psi_{\mathrm{c}}=\frac{2 c_{p \mathrm{a}} T_{\mathrm{amb}}\left(\Pi_{\mathrm{c}}^{1-1 / \gamma_{\mathrm{a}}}-1\right)}{R_{\mathrm{c}}^{2} \omega_{\mathrm{t}}^{2}}
$$

which is the ratio of the isentropic kinetic energy of the gas at the given pressure ratio $\Pi_{\mathrm{c}}$ to the kinetic energy of the compressor blade tip where $R_{\mathrm{c}}$ is the compressor blade radius. The second variable is the volumetric flow coefficient [31]

$$
\Phi_{\mathrm{c}}=\frac{W_{\mathrm{c}} / \rho_{\mathrm{amb}}}{\pi R_{\mathrm{c}}^{3} \omega_{\mathrm{t}}}=\frac{R_{\mathrm{a}} T_{\mathrm{amb}}}{p_{\mathrm{amb}} \pi R_{\mathrm{c}}^{3} \omega_{\mathrm{t}}} W_{\mathrm{c}}
$$

which is the ratio of the volume flowrate of air into the compressor to the rate at which volume is displaced by the compressor blade where $\rho_{\mathrm{amb}}$ is the density of the ambient air. The relation between $\Psi_{\mathrm{c}}$ and $\Phi_{\mathrm{c}}$ can be described by a part of an ellipse $[25,27]$

$$
c_{\Psi 1}\left(\omega_{\mathrm{t}}\right)\left(\Psi_{\mathrm{c}}-c_{\Psi 2}\right)^{2}+c_{\Phi 1}\left(\omega_{\mathrm{t}}\right)\left(\Phi_{\mathrm{c}}-c_{\Phi 2}\right)^{2}=1
$$

where $c_{\Psi 1}$ and $c_{\Phi 1}$ vary with the turbo speed $\omega_{\mathrm{t}}$ and are modelled as the polynomial functions

$$
\begin{aligned}
& c_{\Psi 1}\left(\omega_{\mathrm{t}}\right)=c_{\omega \Psi 1} \omega_{\mathrm{t}}^{2}+c_{\omega \Psi 2} \omega_{\mathrm{t}}+c_{\omega \Psi 3} \\
& c_{\Phi 1}\left(\omega_{\mathrm{t}}\right)=c_{\omega \Phi 1} \omega_{\mathrm{t}}^{2}+c_{\omega \Phi 2} \omega_{\mathrm{t}}+c_{\omega \Phi 3}
\end{aligned}
$$

The mass flow is modelled by solving equation (68) for $\Phi_{\mathrm{c}}$ and solving equation (67) for $W_{\mathrm{c}}$ according to

$$
\begin{aligned}
& \Phi_{\mathrm{c}}=\sqrt{\max \left(0, \frac{1-c_{\Psi 1}\left(\Psi_{\mathrm{c}}-c_{\Psi 2}\right)^{2}}{c_{\Phi 1}}\right)}+c_{\Phi 2} \\
& W_{\mathrm{c}}=\frac{p_{\mathrm{amb}} \pi R_{\mathrm{c}}^{3} \omega_{\mathrm{t}}}{R_{\mathrm{a}} T_{\mathrm{amb}}} \Phi_{\mathrm{c}}
\end{aligned}
$$

Tuning model parameters. The tuning parameters are the parameters $c_{\Psi^{2}}$ and $c_{\Phi 2}$ in the ellipse model for the compressor mass flow, the coefficients $c_{\omega \Psi 1}$, $c_{\omega \Psi 2}$ and $c_{\omega \Psi 3}$ in the polynomial function (69) and the coefficients $c_{\omega \Phi 1}, c_{\omega \Phi 2}$ and $c_{\omega \Phi 3}$ in the polynomial function (70).

Initialization method. The tuning parameters above are initialized by solving a separable nonlinear least-squares problem; see reference [26] for details of the solution method. The non-linear part of this problem minimizes $\left(c_{\Psi_{1}}\left(\omega_{\mathrm{t}}\right)\left(\Psi_{\mathrm{c}}-c_{\Psi_{2}}\right)^{2}+\right.$ $\left.c_{\Phi 1}\left(\omega_{\mathrm{t}}\right)\left(\Phi_{\mathrm{c}}-c_{\Phi 2}\right)^{2}-1\right)^{2}$ with $c_{\Psi 2}$ and $c_{\Phi 2}$ as the optimization variables. In each iteration in the non-linear least-squares solver, the values for $c_{\omega \Psi 1}, c_{\omega \Psi 2}, c_{\omega \Psi 3}$, $c_{\omega \Phi 1}, c_{\omega \Phi 2}$, and $c_{\omega \Phi 3}$ are set to be the solution of a linear least-squares problem that minimizes $\left(c_{\Psi 1}\left(\omega_{\mathrm{t}}\right)\left(\Psi_{\mathrm{c}}-c_{\Psi_{2}}\right)^{2}+c_{\Phi 1}\left(\omega_{\mathrm{t}}\right)\left(\Phi_{\mathrm{c}}-c_{\Phi 2}\right)^{2}-1\right)^{2}$ for the current values of $c_{{ }^{2} 2}$ and $c_{\Phi 2}$. Stationary measurements are used as inputs to the model. The result of the initialization is that the model describes the compressor mass flow well with a mean absolute relative error of 3.4 percent and a maximum absolute relative error of 13.7 percent. The parameters are then tuned according to the method in section 8.1.

\subsubsection{Compressor map}

The compressor performance is usually presented in terms of a map with $\Pi_{\mathrm{c}}$ and $W_{\mathrm{c}}$ on the axes showing the curves of constant efficiency and constant turbo speed. This is shown in Fig. 7 which has approximately the same characteristics as Fig. 2.10 in reference [28]. Consequently, the proposed model of the compressor efficiency equation (62) and the compressor flow equation (72) has the expected behaviour.

\section{INTERCOOLER AND EGR COOLER}

To construct a simple model, that captures the important system properties, the intercooler and the EGR cooler are assumed to be ideal, i.e. there is 
no pressure loss, no mass accumulation, and perfect efficiency, which gives the equations

$$
p_{\text {out }}=p_{\text {in }}, \quad W_{\text {out }}=W_{\text {in }}, \quad T_{\text {out }}=T_{\text {cool }}
$$

where $T_{\text {cool }}$ is the cooling temperature. The model can be extended with non-ideal coolers, but these increase the complexity of the model since nonideal coolers require that there are states for the pressures both before and after the coolers.

\section{MODEL TUNING AND VALIDATION}

One step in the development of a model that describes the system dynamics and the non-linear effects is the tuning and validation. In section 8.1 the model tuning is described, in section 8.2 the importance of different steps in the tuning method is illustrated, and in section 8.3 a validation of the complete model is performed using dynamic data. In the validation, it is important to investigate whether the model captures the essential dynamic behaviours and non-linear effects. The data that are used in the tuning and validation are described in section 2.2.

\subsection{Tuning}

As described in section 2.3, the model parameters are estimated in five steps.

\subsubsection{Initialization method}

First, the parameters in the static models are initialized automatically using least-squares optimization and tuning data from stationary measurements. The initialization methods for each parameter and the results are described in sections 4 to 6 .

\subsubsection{Parameters in dynamic models}

Second, the actuator parameters are estimated using the method in sections 5.2 and 6.2.3. These sections also show the tuning results for the actuators.

Third, the manifold volumes and the turbocharger inertia are estimated by solving the leastsquares optimization problem

$$
\begin{aligned}
& \min V(\theta) \\
& \text { s.t. } \theta_{\min } \leq \theta \leq \theta_{\max }
\end{aligned}
$$

where $V(\theta)$ is the cost function given by

$$
V(\theta)=\sum_{k=1}^{K} \sum_{j=1}^{J} \frac{1}{L_{j}} \sum_{l=1}^{L_{j}}\left(w_{k}^{\text {meas,dyn }}[l]-w_{k}^{\text {mod,dyn }}[l]\right)^{2}
$$

and $\theta$ represents the manifold volumes and the turbocharger inertia, and $\theta_{\min }$ and $\theta_{\max }$ are the lower bound and the upper bound respectively for the parameters. The cost function minimizes the errors between the dynamic measurements $w_{k}^{\text {meas,dyn }}$ and the dynamic simulations $w_{k}^{\text {mod,dyn }}$ of the complete model. There are four outputs $(K=4)$ in the dynamic measurements and simulations, as given by

$$
w_{1}=p_{\mathrm{im}}^{\mathrm{norm}}, \quad w_{2}=p_{\mathrm{em}}^{\mathrm{norm}}, \quad w_{3}=W_{\mathrm{c}}^{\mathrm{norm}}, \quad w_{4}=n_{\mathrm{t}}^{\mathrm{norm}}
$$

where each output and each step response is normalized so that the initial and final values for each step response are the same for both the measurements and the simulations, i.e. there are only dynamic errors in the outputs and no stationary errors.

Further, $J$ is the number of data sets in the dynamic measurements with $J=2$ where the data sets are the sets B and C in Table 1 . The constant $L_{j}$ is the number of samples in data set $j$ where both dynamic measurements and simulations are sampled with a frequency of $100 \mathrm{~Hz}$. The sum $\sum_{l=1}^{L_{j}}$ of each data set in the cost function is normalized with $L_{j}$ in order to penalize each data set equally.

Fourth, the time constant for the engine torque is estimated in the following way. A dynamometer is fitted to the engine via an axle shaft in order to brake or supply torque to the engine. This dynamometer and axle shaft lead to the fact that the measured engine torque has a time constant (see Fig. 19 on page 61 of reference [9]) which is not modelled because the torque will not be used as a feedback in the controller. However, in order to validate the engine torque model during dynamic responses, these dynamics are modelled in the validation as a first-order system

$$
\frac{\mathrm{d}}{\mathrm{d} t} M_{\mathrm{dynamo}}=\frac{1}{\tau_{M_{\mathrm{e}}}}\left(M_{\mathrm{e}}-M_{\text {dynamo }}\right)
$$

where $M_{\text {dynamo }}$ is the torque in the dynamometer and $M_{\mathrm{e}}$ is the output torque from the engine. The time constant $\tau_{M_{\mathrm{e}}}$ is tuned by solving the same optimization problem as in equations (74) and (75) but with $\theta=\tau_{M_{\mathrm{e}}}, K=1$, and $J=1$ with the data set $\mathrm{I}$ in Table $1, w_{1}=M_{\text {dynamo, }}^{\text {norm }}$ and where both dynamic measurements and simulations are sampled with a frequency of $10 \mathrm{~Hz}$.

\subsubsection{Parameters in the static models}

Fifth, the parameters in static models are finally estimated automatically using a least-squares optimization that minimizes errors both in the submodels and in the complete model. This optimization problem is formulated in the same way as in equation (74) but with the cost function 


$$
\begin{aligned}
V(\theta) & =\frac{1}{K J} \sum_{k=1}^{K} \sum_{j=1}^{J} \frac{\gamma_{j}^{2}}{L_{j}} \sum_{l=1}^{L_{j}}\left(\frac{y_{k}^{\text {meas,dyn }}[l]-y_{k}^{\text {mod,dyn }}[l]}{\frac{1}{M} \sum_{m=1}^{M} y_{k}^{\text {meas,stat }}[m]}\right)^{2} \\
& +\frac{1}{\beta N M} \sum_{n=1}^{N} \sum_{p=1}^{M}\left(\frac{z_{n}^{\text {meas,stat }}[p]-z_{n}^{\text {mod,stat }}[p]}{\frac{1}{M} \sum_{m=1}^{M} z_{n}^{\text {meas,stat }}[m]}\right)^{2}
\end{aligned}
$$

where $\theta$ represents the static model parameters.

The first row in the cost function minimizes the errors between dynamic measurements $y_{k}^{\text {meas,dyn }}$ and dynamic simulations $y_{k}^{\text {mod,dyn }}$ of the complete model. The second row minimizes the errors between stationary measurements $z_{n}^{\text {meas,stat }}$ and static submodels $z_{n}^{\text {mod,stat }}$. There are four outputs $(K=4)$ in the dynamic measurements and simulations, namely

$$
y_{1}=p_{\mathrm{im}}, \quad y_{2}=p_{\mathrm{em}}, \quad y_{3}=W_{\mathrm{c}}, \quad y_{4}=n_{\mathrm{t}}
$$

and there are seven outputs $(N=7)$ in the stationary measurements and static submodels, namely

$$
\begin{aligned}
& z_{1}=W_{\mathrm{ei}}, \quad z_{2}=T_{\mathrm{em}}, \quad z_{3}=W_{\mathrm{egr}}, \quad z_{4}=\eta_{\mathrm{tm}}, \\
& z_{5}=W_{\mathrm{t}}, \quad z_{6}=\eta_{\mathrm{c}}, \quad z_{7}=W_{\mathrm{c}}
\end{aligned}
$$

The signals $z_{n}^{\text {meas,stat }}$ and $z_{n}^{\text {mod,stat }}$ are calculated according to the initialization methods in sections 4 to 6 . Further, the number $J$ of data sets in the dynamic measurements is 9 where the data sets are the sets A to I in Table 1. Both the dynamic measurements and the simulations are sampled at a frequency of $10 \mathrm{~Hz}$. The constant $M$ is the number of operating points in the stationary measurements with $M=82$.

Each term in the first row in the cost function is normalized with the mean value of the stationary measurements for the outputs $y_{k}^{\text {meas,stat }}$ and each term in the second row is normalized with the mean value of the stationary measurements for the outputs $z_{n}^{\text {meas,stat }}$. Further, the first row is normalized with $K J$ and the second row is normalized with $N M$ in order penalize dynamic and stationary measurements equally. The parameters $\beta$ and $\gamma_{j}$ are tuning parameters in the optimization problem and they are set to

$$
\beta=1, \quad \gamma_{7}=2, \quad \gamma_{8}=4, \quad \gamma_{j}=1, \text { for } j=1, \ldots, 6,9
$$

The optimization problem is solved using a standard MATLAB local non-linear least-squares solver and the parameters are initialized using the method in the first step above. The result of the optimization is given in Table 2, showing that the mean absolute relative errors are 4.8 per cent or lower.

The calculation time for evaluating the cost function (77) is $42 \mathrm{~s}$ in MATLAB/Simulink on a personal computer with an Intel Core 2 Quad $(2.8 \mathrm{GHz})$, and this cost function was evaluated 190 times when the optimization problem (74) and (77) was solved. Further, the calculation time for simulating the model on the European transient cycle is $28 \mathrm{~s}$, where the cycle is $1800 \mathrm{~s}$ long.

To investigate how much the tuning affects the values of the intake manifold volume $V_{\mathrm{im}}$ and the exhaust manifold volume $V_{\mathrm{em}}$, the physical values of these two parameters are compared with the optimization results. The physical value of $V_{\mathrm{im}}$ is $37 \mathrm{dm}^{3}$ (which also includes the intercooler volume since they are lumped in the model) and the optimization result is $45 \mathrm{dm}^{3}$, i.e. an increase of 22 per cent. The physical value of $V_{\mathrm{em}}$ is $20 \mathrm{dm}^{3}$ and the optimization result is $23 \mathrm{dm}^{3}$, i.e. an increase of 15 per cent. These deviations are reasonable in combination with the fact that they are both increased. The increase can also to some extent be attributed to the additional volumes of fitting pipes as well as the inlets and outlet of neighbouring components

\begin{tabular}{|c|c|c|c|c|c|c|c|c|c|}
\hline \multirow[b]{2}{*}{ Data set } & \multicolumn{8}{|c|}{ VGT and EGR steps } & \multirow{2}{*}{$\begin{array}{l}u_{\delta} \text { steps } \\
\text { I }\end{array}$} \\
\hline & A & B & $\mathrm{C}$ & $\mathrm{D}$ & $\mathrm{E}$ & $\mathrm{F}$ & G & $\mathrm{H}$ & \\
\hline Speed (r/min) & 1200 & 1200 & 1200 & 1200 & 1500 & 1900 & 1900 & 1900 & 1500 \\
\hline Load (\%) & 25 & 40 & 50 & 75 & 50 & 25 & 75 & 100 & - \\
\hline Number of steps & 77 & 23 & 2 & 77 & 77 & 77 & 55 & 1 & 7 \\
\hline$p_{\text {im }}$ & 1.1 & 2.1 & 3.3 & 2.3 & 2.8 & 4.1 & 2.0 & 2.2 & 3.3 \\
\hline$p_{\mathrm{em}}$ & 1.1 & 2.5 & 1.5 & 1.9 & 2.7 & 3.1 & 3.9 & 1.9 & 4.6 \\
\hline$n_{\mathrm{t}}$ & 4.1 & 4.2 & 1.4 & 2.2 & 2.6 & 4.3 & 2.3 & 4.8 & 3.0 \\
\hline$M_{\mathrm{e}}$ & - & - & - & - & - & - & - & - & 4.8 \\
\hline
\end{tabular}
which are not included in the values of the physical components volume but these do contribute to the dynamic response in the lumped-parameter model.

Table 2 The mean absolute relative errors between the dynamic tuning data and diesel engine model simulation where the model parameters are tuned according to the method in section 8.1 
The fuel injection $u_{\delta}$ was not measured in the data sets A to $\mathrm{H}$. In order to obtain values for $u_{\delta}$ for these measurements, $u_{\delta}$ is calculated by inverting the engine torque model (26) to (31), (76) with $u_{\delta}$ as the input and $M_{\text {dynamo }}$ as the output. This means that there will be no errors for the engine torque model. Therefore $M_{\mathrm{e}}$ is not included in the outputs $y_{k}$ and $z_{n}$, the engine torque model parameters are not included in $\theta$, and the errors for the engine torque are not calculated for the data sets $\mathrm{A}$ to $\mathrm{H}$ in Table 2. This will not affect the tuning of the other model parameters since the engine torque does not affect the outputs $y_{k}$ and $z_{n}$.

\subsection{The importance of the initialization method and the cost function (77)}

To illustrate the importance of the initialization method in the first step in the proposed tuning method and the cost function (77), the tuning method in section 8.1 is compared with two other tuning methods.

First, if each parameter $\theta(i)$ in the static models is initialized to

$$
\theta(i)=\left\{\begin{array}{cll}
\theta_{\max }(i) & \text { if } & \theta_{\max }(i)<1 \\
1 & \text { if } & \theta_{\min }(i) \leq 1 \leq \theta_{\max }(i) \\
\theta_{\min }(i) & \text { if } & \theta_{\min }(i)>1
\end{array}\right.
$$

and if only the first row is used in equation (77), i.e. the complete model (which is a common choice of cost function in system identification), the mean absolute relative errors become 480 percent or lower when a local solver is applied. This high error arises because the optimization is non-convex with at least two local minima. Consequently, by optimizing the sub-models in the initialization a simple local solver can be used for the non-convex optimization problem to obtain small errors in the complete model. It is also important to use the submodels in the cost function to guarantee that each submodel describes the measurements well.

Second, if only the first four steps in the tuning method are used (which is a common choice of tuning method for mean value engine models), the mean absolute relative errors become 12.7 percent or lower, compared with 4.8 percent or lower for the proposed tuning method. Consequently, it is important to use both the submodels and the complete model in the optimization and not only the submodels.

\subsection{Validation}

Because there are few stationary measurements, both the static and the dynamic models are validated by simulating the total model and comparing it with the dynamic validation data sets $\mathrm{Q}$ to $\mathrm{V}$ in Table 1 . The result of this validation can be seen in Table 3 which shows that the mean absolute relative errors are 5.8 percent or lower. The relative errors are due mostly to steady state errors but, since the engine model will be used in a controller, the steady state accuracy is less important since a controller will take care of steady state errors. However, in order to design a successful controller, it is important that the model captures the main steady state behaviours, essential dynamic behaviours, and non-linear effects. Therefore, essential system properties and time constants are validated in the following section.

\subsubsection{Validation of essential system properties and time constants}

References [2] and [3] show the essential system properties for the pressures and the flows in a diesel engine with VGT and EGR. Some of these properties are a non-minimum phase behaviour in the intake manifold pressure and a non-minimum

Table 3 The mean absolute relative errors between dynamic validation data and diesel engine model simulation where the model parameters are tuned according to the method in section 8.1 (mean value of all data sets and outputs, 3.3 per cent)

\begin{tabular}{|c|c|c|c|c|c|c|}
\hline \multirow[b]{2}{*}{ Data set } & \multicolumn{5}{|c|}{ VGT and EGR steps } & \multirow{2}{*}{$\begin{array}{l}u_{\delta} \text { steps } \\
\mathrm{V}\end{array}$} \\
\hline & $\mathrm{Q}$ & $\mathrm{R}$ & $\mathrm{S}$ & $\mathrm{T}$ & U & \\
\hline Speed (r/min) & 1200 & 1200 & 1500 & 1500 & 1900 & 1500 \\
\hline Load (\%) & 40 & 50 & 25 & 75 & 50 & - \\
\hline Number of steps & 12 & 77 & 77 & 77 & 77 & 28 \\
\hline$p_{\text {im }}$ & 2.4 & 2.5 & 1.9 & 3.8 & 2.3 & 4.6 \\
\hline$p_{\mathrm{em}}$ & 2.2 & 2.0 & 1.9 & 3.8 & 3.4 & 5.8 \\
\hline$W_{\mathrm{c}}$ & 3.2 & 3.7 & 2.2 & 5.8 & 4.5 & 4.0 \\
\hline$n_{\mathrm{t}}$ & 4.0 & 2.4 & 2.2 & 3.7 & 3.4 & 4.4 \\
\hline$M_{\mathrm{e}}$ & - & - & - & - & - & 3.5 \\
\hline
\end{tabular}



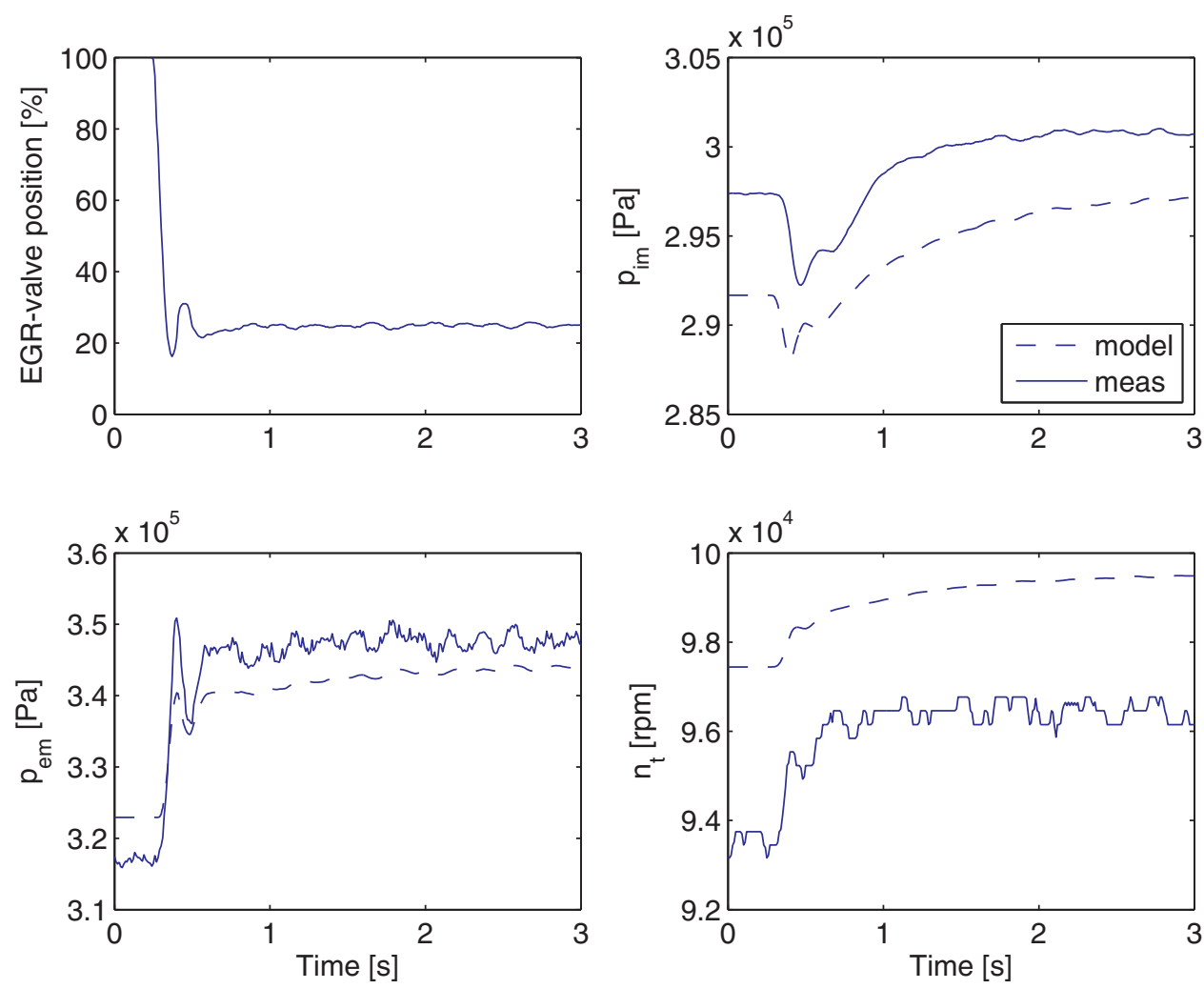

Fig. 8 Comparison between diesel engine model simulation and dynamic validation data during a step in the EGR-valve position showing that the model captures the non-minimum phase behaviour in $p_{\text {im }}$ (data set $\mathrm{H}$; operating point; 100 per cent load, $n_{\mathrm{e}}=1200 \mathrm{r} / \mathrm{min}$, and $u_{\mathrm{vgt}}=60$ per cent

phase behaviour, an overshoot, and a sign reversal in the compressor mass flow. These system properties and time constants are validated using the dynamic validation data sets $\mathrm{A}, \mathrm{D}$ to $\mathrm{I}$, and $\mathrm{Q}$ to $\mathrm{V}$ in Table 1. Three validations are performed in Figs 8 to 10. Figure 8 shows that the model captures the non-minimum phase behaviour in the channel $u_{\text {egr }}$ to $p_{\text {im }}$. Figure 9 shows that the model captures the non-minimum phase behaviour in the channel $u_{\mathrm{vgt}}$ to $W_{\mathrm{c}}$. Figure 10 shows that the model captures the overshoot in the channel $u_{\mathrm{vgt}}$ to $W_{\mathrm{c}}$ and a small non-minimum phase behaviour in the channel $u_{\mathrm{vgt}}$ to $n_{\mathrm{t}}$. Figures 8 to 10 also show that the model captures the fast dynamics at the beginning of the responses and the slow dynamics at the end of the responses. Further, by comparing Figs 9 and 10, it can be seen that the model captures the sign reversal in $u_{\text {vgt }}$ to $W_{\mathrm{c}}$. In Fig. 9 the d.c. gain is negative and in Fig. 10 the d.c. gain is positive.

\section{MODEL EXTENSIONS}

The proposed model in section 3 to 8 is a small model with eight states that describe the
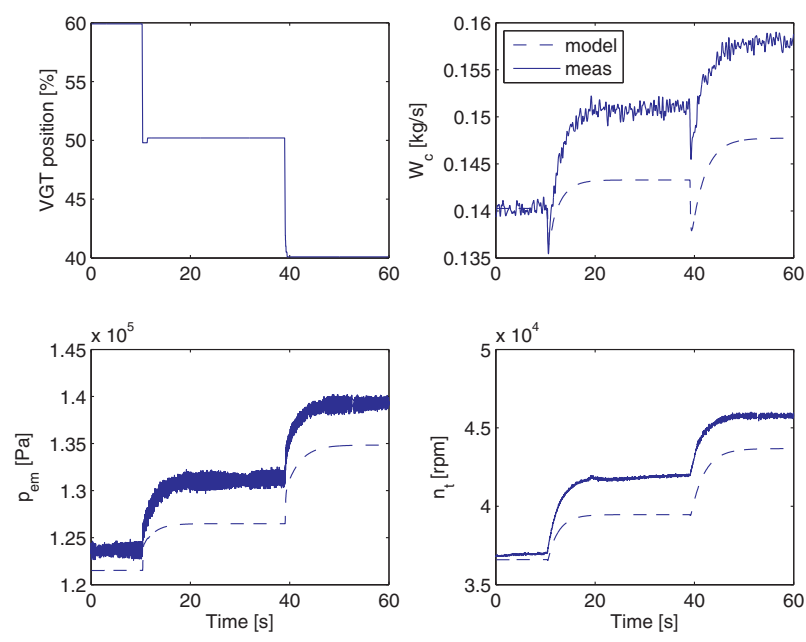

Fig. 9 Comparison between diesel engine model simulation and dynamic validation data during steps in the VGT position showing that the model captures the non-minimum phase behaviour in $W_{\mathrm{c}}$ (data set Q; operating point; 40 percent load, $n_{\mathrm{e}}=1200 \mathrm{r} / \mathrm{min}$, and $u_{\mathrm{egr}}=100$ per cent)

important dynamics and non-linear system properties according to section 8. In the following sections the goal is to investigate whether this model 

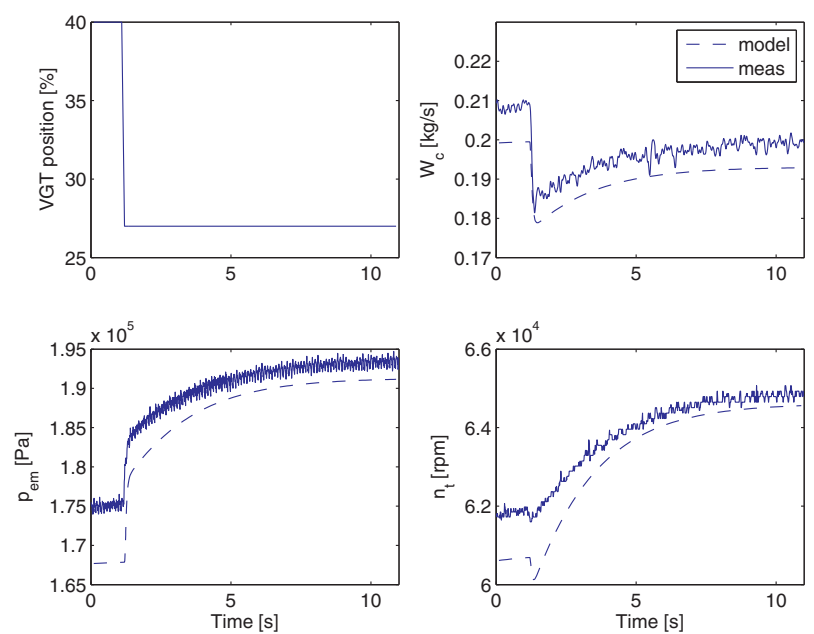

Fig. 10 Comparison between diesel engine model simulation and dynamic tuning data during a step in the VGT position showing that the model captures the overshoot in $W_{\mathrm{c}}$ and a small nonminimum phase behaviour in $n_{\mathrm{t}}$; a comparison between Figs 9 and 10 also shows that the model captures the sign reversal in $W_{\mathrm{c}}$ (data set C; operating point; 50 percent load, $n_{\mathrm{e}}=1200 \mathrm{r} / \mathrm{min}$ and $u_{\text {egr }}=100$ per cent)

can be improved substantially with relatively small model extensions. In section 9.1 the proposed model is extended with temperature states and in section 9.2 the proposed model is extended with temperature states, a pressure drop over the intercooler, and an extra control volume. The main goal is to investigate whether the dynamic behaviour can be improved, but it is also interesting to investigate whether the steady state behaviour can be improved substantially since this might improve the feedforward part of a controller that is based on the extended model.

\subsection{Extensions: temperature states}

To investigate whether temperature states in the manifolds improve the model substantially, the eighth-order model in sections 3 to 7 is extended with two temperature states $T_{\mathrm{im}}$ and $T_{\mathrm{em}}$, which leads to a tenth-order model with the states

$$
\begin{aligned}
& \boldsymbol{x}=\left(\begin{array}{llllll}
p_{\mathrm{im}} & p_{\mathrm{em}} & T_{\mathrm{im}} & T_{\mathrm{em}} & X_{\mathrm{Oim}} & X_{\text {Oem }}
\end{array}\right. \\
& \begin{array}{llll}
\omega_{\mathrm{t}} & \tilde{u}_{\text {egrl }} & \tilde{u}_{\text {egr2 }} & \left.\tilde{u}_{\text {vgt }}\right)^{\mathrm{T}}
\end{array}
\end{aligned}
$$

\subsubsection{Extended model equations}

The intake and exhaust manifold models in section 3 are extended with temperatures states $T_{\mathrm{im}}$ and $T_{\mathrm{em}}$ according to the adiabatic model $[\mathbf{3 2}, \mathbf{3 3}]$

$$
\begin{aligned}
& \frac{\mathrm{d}}{\mathrm{d} t} T_{\mathrm{im}}=\frac{R_{\mathrm{a}} T_{\mathrm{im}}}{p_{\mathrm{im}} V_{\mathrm{im}} c_{V \mathrm{a}}}\left\{c_{V \mathrm{a}}\left(W_{\mathrm{ic}}+W_{\text {egr }}\right)\left(T_{\mathrm{im}, \text { in }}-T_{\mathrm{im}}\right)\right. \\
& \left.\left.+R_{\mathrm{a}}\left[T_{\mathrm{im}, \text { in }}\left(W_{\mathrm{ic}}+W_{\text {egr }}\right)-T_{\mathrm{im}} W_{\text {ei }}\right)\right]\right\} \\
& \frac{\mathrm{d}}{\mathrm{d} t} p_{\mathrm{im}}=\frac{R_{\mathrm{a}} T_{\mathrm{im}}}{V_{\mathrm{im}}}\left(W_{\mathrm{ic}}+W_{\text {egr }}-W_{\mathrm{ei}}\right)+\frac{p_{\mathrm{im}}}{T_{\mathrm{im}}} \frac{\mathrm{d}}{\mathrm{d} t} T_{\mathrm{im}} \\
& \frac{\mathrm{d}}{\mathrm{d} t} T_{\mathrm{em}}=\frac{R_{\mathrm{e}} T_{\mathrm{em}}}{p_{\mathrm{em}} V_{\mathrm{em}} c_{V \mathrm{e}}}\left\{c_{V \mathrm{e}} W_{\mathrm{eo}}\left(T_{\mathrm{em}, \mathrm{in}}-T_{\mathrm{em}}\right)\right. \\
& \left.+R_{\mathrm{e}}\left[T_{\mathrm{em}, \text { in }} W_{\text {eo }}-T_{\mathrm{em}}\left(W_{\mathrm{t}}+W_{\mathrm{egr}}\right)\right]\right\} \\
& \frac{\mathrm{d}}{\mathrm{d} t} p_{\mathrm{em}}=\frac{R_{\mathrm{e}} T_{\mathrm{em}}}{V_{\mathrm{em}}}\left(W_{\mathrm{eo}}-W_{\mathrm{t}}-W_{\mathrm{egr}}\right)+\frac{p_{\mathrm{em}}}{T_{\mathrm{em}}} \frac{\mathrm{d}}{d \mathrm{t}} T_{\mathrm{em}}(81)
\end{aligned}
$$

where the temperature $T_{\mathrm{im} \text {,in }}$ for the flows into the intake manifold is assumed to be constant and the temperature $T_{\mathrm{em}, \text { in }}$ for the flow into the exhaust manifold is equal to $T_{\mathrm{em}}$ in equation (25). The intercooler is assumed to be ideal, and thus

$$
W_{\mathrm{ic}}=W_{\mathrm{c}}
$$

The differential equations for the oxygen mass fractions are the same as in section 3 if equation (82) is applied to

$$
\begin{aligned}
\frac{\mathrm{d}}{\mathrm{d} t} X_{\text {Oim }}= & \frac{R_{\mathrm{a}} T_{\mathrm{im}}}{p_{\mathrm{im}} V_{\text {im }}}\left[\left(X_{\mathrm{Oem}}-X_{\text {Oim }}\right) W_{\mathrm{egr}}+\right. \\
& \left.\left(X_{\mathrm{Oc}}-X_{\text {Oim }}\right) W_{\mathrm{ic}}\right] \\
\frac{\mathrm{d}}{\mathrm{d} t} X_{\mathrm{Oem}}= & \frac{R_{\mathrm{e}} T_{\mathrm{em}}}{p_{\mathrm{em}} V_{\mathrm{em}}}\left(X_{\mathrm{Oe}}-X_{\mathrm{Oem}}\right) W_{\mathrm{eo}}
\end{aligned}
$$

The values of all the tuning parameters are the same as for the eighth-order model.

\subsubsection{Comparison between the eighth-and the tenth- order models}

To investigate how the states $T_{\mathrm{im}}$ and $T_{\mathrm{em}}$ affect the system properties, step responses are compared for the eighth- and tenth-order model. Figures 11 and 12 show that the two models have approximately the same dynamic responses with approximately the same non-minimum phase behaviours, overshoots, and sign reversals in $W_{\mathrm{c}}$. Consequently, the temperature states have only minor effects on the system properties and therefore there are no major improvements in the model if it is extended with temperature states.

\subsection{Extensions: temperature states and pressure drop over the intercooler}

To investigate whether additional temperature states and a pressure drop over the intercooler improve the model substantially, the tenth-order model in section 9.1 is extended with a control 

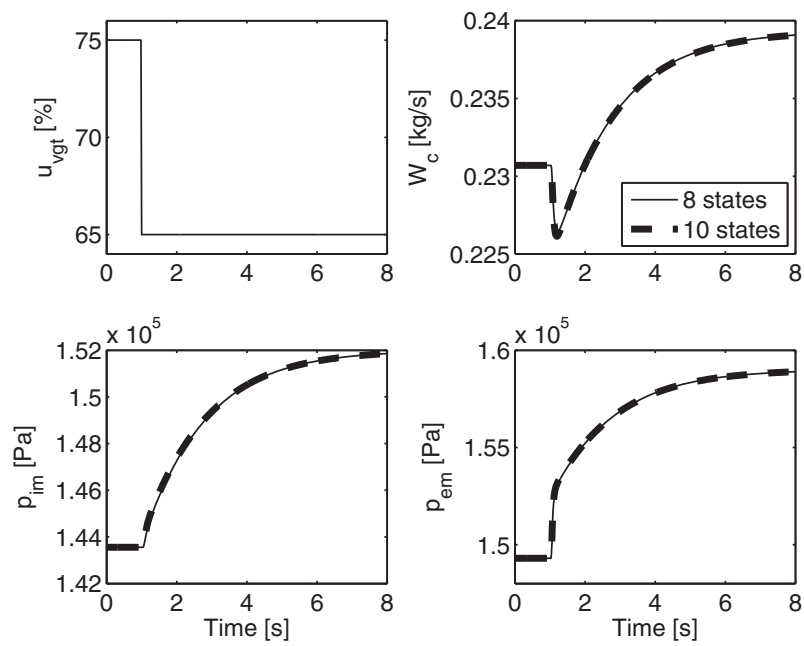

Fig. 11 Comparison between the eighth- and the tenth-order models during a step in the VGT position, showing that these two models have approximately the same dynamic responses with non-minimum phase behaviour in $W_{\mathrm{c}}$ (operating point, $u_{\delta}=110 \mathrm{mg} /$ cycle, $n_{\mathrm{e}}=1500$ $\mathrm{r} / \mathrm{min}$, and $u_{\text {egr }}=80$ per cent)
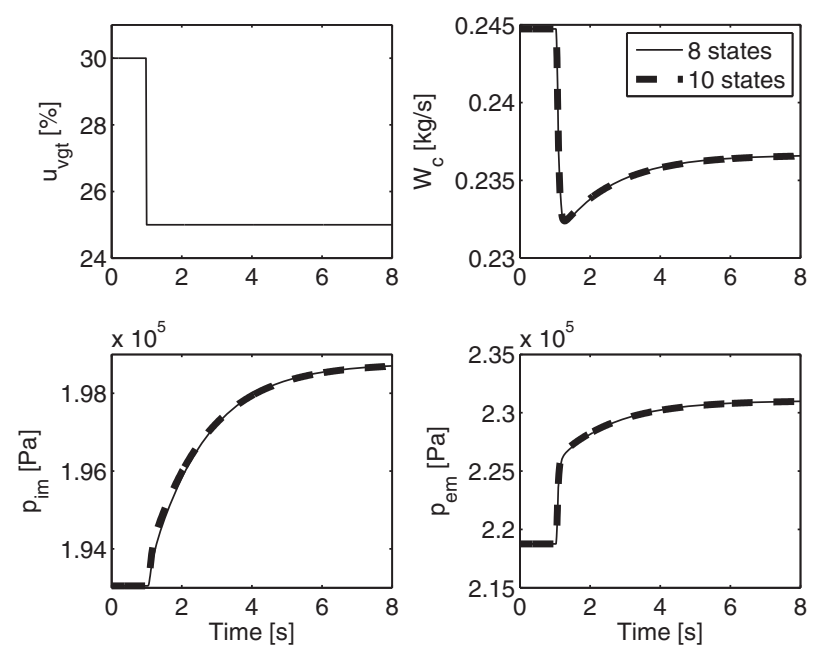

Fig. 12 Comparison between the eighth- and the tenth-order models during a step in the VGT position showing that these two models have approximately the same dynamic responses with an overshoot and a sign reversal in $W_{\mathrm{c}}$ (operating point, $u_{\delta}=110 \mathrm{mg} /$ cycle, $n_{\mathrm{e}}=1500$ $\mathrm{r} / \mathrm{min}$, and $u_{\text {egr }}=80$ per cent)

volume between the compressor and the intercooler. This control volume consists of a temperature state $T_{\mathrm{ic}}$ and a pressure state $p_{\mathrm{c}}$. This leads to a twelfth-order model with the states

$$
\begin{aligned}
\boldsymbol{x}=\left(\begin{array}{lllllll}
p_{\mathrm{im}} & p_{\mathrm{em}} & p_{\mathrm{c}} & T_{\mathrm{im}} & T_{\mathrm{em}} & T_{\mathrm{ic}} \\
X_{\text {Oim }} & X_{\text {Oem }} & \omega_{\mathrm{t}} & \tilde{u}_{\text {egr } 1} & \tilde{u}_{\text {egr2 }} & \tilde{u}_{\text {vgt }}
\end{array}\right)^{\mathrm{T}}
\end{aligned}
$$

\subsubsection{Extended model equations}

The control volume before the intercooler is modelled as an adiabatic model with a temperature state $T_{\text {ic }}$ and a pressure state $p_{\mathrm{c}}$ according to

$$
\begin{aligned}
\frac{\mathrm{d}}{\mathrm{d} t} T_{\mathrm{ic}}= & \frac{R_{\mathrm{a}} T_{\mathrm{ic}}}{p_{\mathrm{c}} V_{\text {ic }} c_{V \mathrm{a}}}\left[c_{V \mathrm{a}} W_{\mathrm{c}}\left(T_{\mathrm{c}}-T_{\mathrm{ic}}\right)\right. \\
& \left.+R_{\mathrm{a}}\left(T_{\mathrm{c}} W_{\mathrm{c}}-T_{\mathrm{ic}} W_{\text {ic }}\right)\right] \\
\frac{\mathrm{d}}{\mathrm{d} t} p_{\mathrm{c}}= & \frac{R_{\mathrm{a}} T_{\mathrm{ic}}}{V_{\mathrm{ic}}}\left(W_{\mathrm{c}}-W_{\mathrm{ic}}\right)+\frac{p_{\mathrm{c}}}{T_{\mathrm{ic}}} \frac{\mathrm{d}}{\mathrm{d} t} T_{\mathrm{ic}}
\end{aligned}
$$

where $V_{\text {ic }}$ is the volume of the control volume. The flow $W_{\text {ic }}$ through the intercooler is modelled as an incompressible flow $[\mathbf{2 8}, \mathbf{2 9}]$

$$
W_{\mathrm{ic}}=\sqrt{\frac{p_{\mathrm{c}}\left(p_{\mathrm{c}}-p_{\mathrm{im}}\right)}{T_{\mathrm{ic}} k_{\mathrm{ic}}}}
$$

Equation (86) is used instead of equation (82) and the pressure ratio over the compressor given by

$$
\Pi_{\mathrm{c}}=\frac{p_{\mathrm{c}}}{p_{\mathrm{amb}}}
$$

is used instead of equation (59).

Tuning parameters for the extended model equations. The parameters are the intercooler volume $V_{\text {ic }}$ and the parameter $k_{\text {ic }}$ for the model in equation (86).

Tuning method. All the tuning parameters in the twelfth-order model are tuned in three steps. First, the tuning parameter $k_{\text {ic }}$ is initialized by solving a linear least-squares problem that minimizes $\left[p_{\mathrm{c}}-\right.$ $\left.p_{\text {im }}-\left(p_{\text {c,meas }}-p_{\text {im,meas }}\right)\right]^{2}$ with $k_{\text {ic }}$ as the optimization variable. The model of $p_{\mathrm{c}}-p_{\mathrm{im}}$ is obtained by solving equation (86) for $p_{\mathrm{c}}-p_{\mathrm{im}}$. The variables $P_{\mathrm{c} \text {,meas }}$ and $P_{\mathrm{im} \text {,meas }}$ are stationary measurements. The initial values of the other parameters in the static models are the same as for the final values of the tuning of the eighth-order model. Second, the intercooler volume $V_{\mathrm{ic}}$, the intake manifold volume $V_{\mathrm{im}}$, the exhaust manifold volume $V_{\mathrm{em}}$, and the turbo inertia $J_{\mathrm{t}}$ are tuned using the same optimization problem as in equations (74) and (75) but with $V_{\text {ic }}$ as an additional parameter in $\theta$. The values of the other parameters in the dynamic models are the same as for the eighth-order model. Third, the parameters in static models are finally estimated automatically using the same least-squares optimization problem as in equations (74) and (77) but with $k_{\text {ic }}$ as an additional parameter in $\theta, N=8, z_{8}=W_{\mathrm{ic}}$, and $\beta=2.58$. 

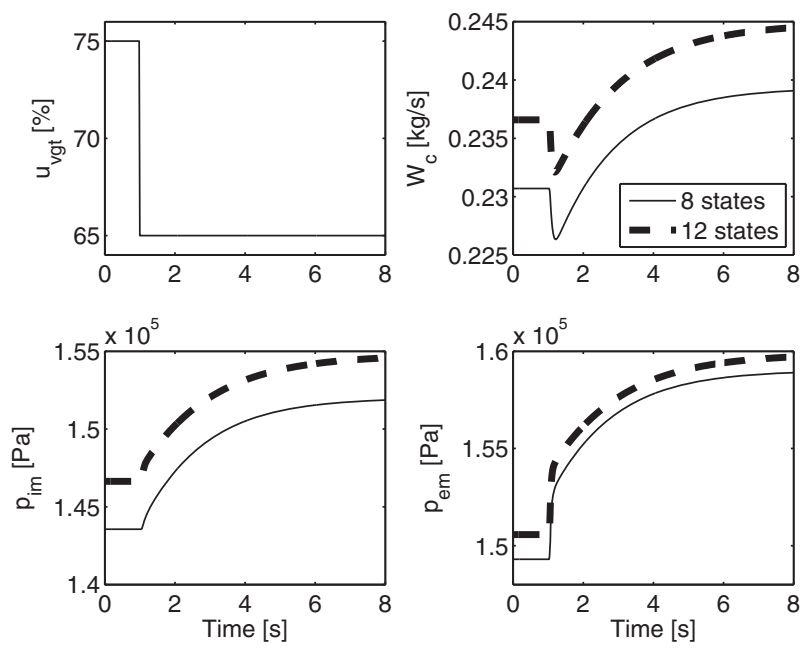

Fig. 13 Comparison between the eighth- and the twelfth-order models during a step in the VGT position, showing that there are stationary differences; however, the dynamic behaviour are approximately the same with a non-minimum phase behaviour in $W_{\mathrm{c}}$ (operating point, $u_{\delta}=$ $110 \mathrm{mg} /$ cycle, $n_{\mathrm{e}}=1500 \mathrm{r} / \mathrm{min}$, and $u_{\mathrm{egr}}=80$ per cent)

\subsubsection{Comparison between the eighth-and twelfth-order models}

To investigate how the additional temperature states, the control volume before the intercooler, and the pressure drop over the intercooler affect the system properties, step responses are compared for the eighth- and twelfth-order models. Figures 13 and 14 show that there are stationary differences between the two models. However, the dynamic behaviours are qualitatively the same considering the amplitudes, the time constants, the nonminimum phase behaviour, the overshoot, and the sign reversals in $W_{\mathrm{c}}$. Therefore, the conclusion is that temperature states, a pressure drop over the intercooler, and a control volume before the intercooler have only small effects on the dynamic behaviour but the addition of the pressure drop does have an effect on the stationary values.

\subsubsection{Comparison between experimental data and the twelfth-order model}

The previous section shows that there are stationary differences between the eighth- and twelfth-order models. In this section, the goal is to investigate whether these stationary differences improve the validation results in section 8.3.

The twelfth-order model is validated by calculating the mean absolute relative errors between the twelfth-order model and dynamic validation data
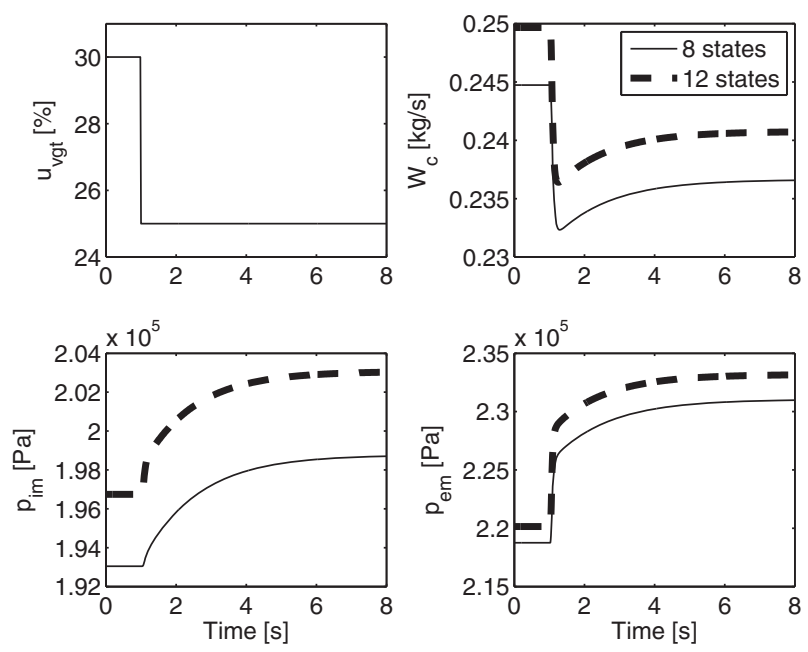

Fig. 14 Comparison between eighth- and twelfthorder models during a step in the VGT position, showing that there are stationary differences; however, the dynamic behaviours are approximately the same with an overshoot and a sign reversal in $W_{\mathrm{c}}$ (operating point, $u_{\delta}=110 \mathrm{mg} /$ cycle, $n_{\mathrm{e}}=1500 \mathrm{r} / \mathrm{min}$, and $u_{\mathrm{egr}}=$ 80 per cent)

(Table 4). These mean absolute relative errors are calculated in the same way as for the eighth-order model in Table 3. Comparing these two tables, there are only small differences between the mean relative errors. The twelfth-order model gives smaller errors at some operating points and larger errors at other operating points. The mean value of all data sets and outputs is 3.5 per cent for the twelfthorder model and 3.3 percent for the eighth-order model. Consequently, the inclusion of temperature states, a pressure drop over the intercooler, and a control volume before the intercooler did not improve the model quality on the validation data.

\section{CONCLUSIONS}

A mean value model of a diesel engine with VGT and EGR is developed and validated. The intended applications of the model are system analysis, simulation, and development of model-based control systems. The goal is to construct a model that describes the dynamics in the manifold pressures, turbocharger, EGR, and actuators with few states in order to obtain short simulation times. Therefore the model has only eight states: the intake and exhaust manifold pressures, the oxygen mass fractions in the intake and exhaust manifolds, the turbocharger speed, and three states describing the actuator dynamics. A Simulink implementation 
Table 4 The mean absolute relative errors between dynamic validation data and simulation of the twelfth-order model (mean value of all data sets and outputs, 3.5 per cent)

\begin{tabular}{|c|c|c|c|c|c|c|}
\hline \multirow[b]{2}{*}{ Data set } & \multicolumn{5}{|c|}{ VGT and EGR steps } & \multirow{2}{*}{$\begin{array}{l}u_{\boldsymbol{\delta}} \text { steps } \\
\mathrm{V}\end{array}$} \\
\hline & Q & $\mathrm{R}$ & $\mathrm{S}$ & $\mathrm{T}$ & $\mathrm{U}$ & \\
\hline Speed (r/min) & 1200 & 1200 & 1500 & 1500 & 1900 & 1500 \\
\hline Load $(\%)$ & 40 & 50 & 25 & 75 & 50 & - \\
\hline Number of steps & 12 & 77 & 77 & 77 & 77 & 28 \\
\hline$p_{\mathrm{im}}$ & 2.6 & 2.3 & 1.8 & 3.6 & 3.3 & 5.1 \\
\hline$p_{\mathrm{em}}$ & 2.9 & 2.0 & 2.3 & 3.7 & 4.2 & 5.9 \\
\hline$n_{\mathrm{t}}$ & 4.3 & 3.0 & 2.6 & 4.0 & 4.1 & 3.4 \\
\hline$M_{\mathrm{e}}$ & - & - & - & - & - & 3.6 \\
\hline
\end{tabular}

including a complete set of parameters of the model are available for download [5].

Many models in the literature, which have approximately the same complexities as the model proposed here, use three states for each control volume in order to describe the temperature dynamics. However, the model proposed here uses only two states for each manifold. Model extensions are investigated showing that inclusion of the temperature states and pressure drop over the intercooler has only minor effects on the dynamic behaviour and does not improve the dynamic responses. Therefore, these extensions are not included in the proposed model. In addition, the cylinder-out temperature is modelled using a fixedpoint iteration and it is shown that it is sufficient to use one iteration in this iterative process to achieve an accuracy of 0.15 per cent.

Model equations and parameters are described for each subsystem in the model. In order to have a small number of tuning parameters, the flows and efficiencies are modelled using physical relationships and parametric models instead of look-up tables. All the model parameters are tuned automatically using several different least-squares optimizations. In the optimization of the parameters in the static models it was shown that it is important to tune both the submodels and the complete model and not only the submodels or not only the complete model.

Static and dynamic validations of the entire model were performed using dynamic measurements, consisting of steps in the fuel injection, EGR, and VGT position. The validations show that the mean relative errors are 5.8 percent or lower for all measured variables. They also show that the proposed model captures the essential system properties, i.e. a non-minimum phase behaviour in the channel $u_{\text {egr }}$ to $p_{\text {im }}$ and a non-minimum phase behaviour, an overshoot, and a sign reversal in the channel $u_{\mathrm{vgt}}$ to $W_{\mathrm{c}}$.

\section{ACKNOWLEDGEMENTS}

This research was supported by the VINNOVA Industry Excellence Center LINK-SIC, by the Linnaeus Center CADICS, funded by the Swedish Research Council, and by Scania CV AB.

(C) Authors 2011

\section{REFERENCES}

1 Heywood, J. B. Internal combustion engine fundamentals, 1988 (McGraw-Hill, New York).

2 Kolmanovsky, I. V., Stefanopoulou, A. G., Moraal, P. E., and van Nieuwstadt, M. Issues in modeling and control of intake flow in variable geometry turbocharged engines. In Proceedings of 18th IFIP Conference on System modeling and optimization, Detroit, Michigan, USA, July 1997, 1998, pp. 436-445 (Addison-Wesley Longman, Harlow, Essex).

3 Jung, M. Mean-value modelling and robust control of the airpath of a turbocharged diesel engine. $\mathrm{PhD}$ Thesis, University of Cambridge, Cambridge, UK, 2003.

4 Vigild, C. The internal combustion engine: modelling, estimation and control issues. $\mathrm{PhD}$ Thesis, Technical University of Denmark, Lyngby, Denmark, 2001.

5 Software packages from Vehicular Systems, 2010, available from http://www.fs.isy.liu.se/Software.

6 Höckerdal, E., Frisk, E., and Eriksson, L. Observer design and model augmentation for bias compensation with a truck engine application. Control Engng Practice, 2009, 17(3), 408-417.

7 Fröberg, A. and Nielsen, L. Efficient drive cycle simulation. IEEE Trans. Veh. Technol., 2008, 57(2), 1442-1453.

8 Wahlström, J., Eriksson, L., and Nielsen, L. EGRVGT control and tuning for pumping work minimization and emission control. IEEE Trans. Control Systems Technol., 2010, 18(4), 993-1003.

9 Wahlström, J. Control of EGR and VGT for emission control and pumping work minimization in diesel engines. PhD Thesis, Linköping University, Linköping, Sweden, 2009. 
10 Eriksson, L., Wahlström, J., and Klein, M. Physical modeling of turbocharged engines and parameter identification. In Automotive model predictive control: models, methods and applications (Eds L. del Re, F. Allgöwer, L. Glielmo, C. Guardiola, and I. Kolmanovsky), 2009, pp. 59-79. (Springer, Betin).

11 Olsson, J. and Welander, M. Optimal control of a diesel engine with VGT and EGR. Master's Thesis, Linköping University, Linköping, Sweden, 2006.

12 Johansson, T. System analysis of a diesel engine with VGT and EGR. Master's Thesis, Linköping University, Linköping, Sweden, 2005.

13 Bergström, C. and Höckerdal, G. Model based diagnosis of the intake manifold pressure on a diesel engine. Master's Thesis, Linköping University, Linköping, Sweden, 2009.

14 Dagsson, J. and Källström, S. N. Air leakage diagnosis in heavy duty truck engines with EGR and VGT. Master's Thesis, Linköping University, Linköping, Sweden, 2009.

15 Nilsson, F. Diagnosis of a truck engine using nonlinear filtering techniques. Master's Thesis, Linköping University, Linköping, Sweden, 2007.

16 Hendricks, E. A compact, comprehensive model of large turbocharged, two-stroke diesel engines. SAE paper 861190, 1986.

17 Watson, N. Dynamic turbocharged diesel engine simulator for electronic control system development. Trans. ASME, Dynamic Systems Measmt Control, 1984, 106, 27-45.

18 Jankovic, M. and Kolmanovsky, I. V. Constructive Lyapunov control design for turbocharged diesel engines. IEEE Trans. Control Systems Technol., 2000, 8(2), 288-299.

19 Ammann, M., Fekete, N. P., Guzzella, L., and Glattfelder, A. H. Model-based control of the VGT and EGR in a turbocharged common-rail diesel engine: theory and passenger car implementation. SAE paper 2003-01-0357, 2003.

20 Stefanopoulou, A. G., Kolmanovsky, I., and Freudenberg, J. Control of variable geometry turbocharged diesel engines for reduced emissions. IEEE Trans. Control Systems Technol., 2000, 8(4), 733-745.

21 Nieuwstadt, M., Kolmanovsky, I. V., Moraal, P. E., Stefanopoulou, A., and Jankovic, M. EGR-VGT control schemes: experimental comparison for a high-speed diesel engine. IEEE Control Systems Mag., 2000, 20(3), 63-79.

22 Rajamani, R. Control of a variable-geometry turbocharged and wastegated diesel engine. Proc. IMechE, Part D: J. Automobile Engineering, 2005, 219(11), 1361-1368. DOI: 10.1243/0954407805X34964.

23 Skogtjärn, P. Modelling of the exhaust gas temperature for diesel engines. Master's Thesis LiTH-ISYEX-3379, Department of Electrical Engineering, Linköping University, Linköping, Sweden, 2002.

24 Eriksson, L. Mean value models for exhaust system temperatures. SAE paper 2002-01-0374, 2002.

25 Andersson, P. Air charge estimation in turbocharged spark ignition engines. $\mathrm{PhD}$ Thesis, Linköping University, Sweden, 2005.
26 Björk, Å. Numerical methods for least squares problems, 1996 (SIAM, Philadelphia, Pennsylvania, USA).

27 Eriksson, L. Modeling and control of turbocharged SI and DI engines. Oil Gas Sci. Technol. - Rev. IFP, 2007, 62(4), 523-538.

28 Watson, N. and Janota, M. S. Turbocharging the internal combustion engine, 1982 (Mechanical Press Ltd, Hong Kong).

29 Eriksson, L., Nielsen, L., Brugård, J., Bergström, J. M., Pettersson, F., and Andersson, P. Modeling and simulation of a turbo charged SI engine. Ann. Rev. Control, 2002, 26(1), 129-137.

30 Guzzella, L. and Amstutz, A. Control of diesel engines. IEEE Control Systems Mag., 1998, 18, 53-71.

31 Dixon, S. L. Fluid mechanics and thermodynamics of turbomachinery, 4th edition, 1998 (ButterworthHeinemann, Oxford).

32 Chevalier, A., Müeller, M., and Hendricks, E. On the validity of mean value engine models during transient operation. SAE paper 2000-011261, 2000.

33 Hendricks, E. Isothermal vs. adiabatic mean value SI engine models. In Proceedings of the Third IFAC Workshop on Advances in automotive control, Karlsruhe, Germany, 28-30 March 2001, pp. 363368 (Pergaman, Oxford).

\section{APPENDIX 1}

\section{Notation}

A

BSR

$c_{p}$

$c_{v}$

$J$

M

$M_{\mathrm{e}}$

$M_{\mathrm{p}}$

$n_{\text {cyl }}$

$n_{\mathrm{e}}$

$n_{\mathrm{t}}$

$(\mathrm{O} / \mathrm{F}) \mathrm{s}$

p

$P$

$q_{\mathrm{HV}}$

$r_{\mathrm{c}}$

$R$

R

$T$

$u_{\text {egr }}$

$u_{\mathrm{vgt}}$

$u_{\delta}$ area $\left(\mathrm{m}^{2}\right)$

blade speed ratio

specific heat capacity at constant pressure $(\mathrm{J} / \mathrm{kg} \mathrm{K})$

specific heat capacity at constant volume $(\mathrm{J} / \mathrm{kg} \mathrm{K})$

inertia $\left(\mathrm{kg} \mathrm{m}^{2}\right)$

torque $(\mathrm{Nm})$

engine torque $(\mathrm{Nm})$

pumping torque (Nm)

number of cylinders

rotational engine speed (r/min)

rotational turbine speed ( $\mathrm{r} / \mathrm{min})$

stoichiometric oxygen-to-fuel ratio

pressure $(\mathrm{Pa})$

power (W)

heating value of fuel $(\mathrm{J} / \mathrm{kg})$

compression ratio

gas constant $(\mathrm{J} / \mathrm{kg} \mathrm{K})$

radius $(\mathrm{m})$

temperature $(\mathrm{K})$

exhaust gas recirculation control signal (100, open; 0 , closed) (\%)

variable-geometry turbocharger control signal (100, open; 0 , closed) (\%)

injected amount of fuel (mg/cycle) 


$\begin{array}{ll}V & \text { volume }\left(\mathrm{m}^{3}\right) \\ W & \text { mass flow }(\mathrm{kg} / \mathrm{s}) \\ x_{\mathrm{egr}} & \text { exhaust gas recirculation fraction } \\ X_{\mathrm{O}} & \text { oxygen mass fraction } \\ \gamma & \text { specific heat capacity ratio } \\ \eta & \text { efficiency } \\ \lambda_{\mathrm{O}} & \text { oxygen-to-fuel ratio } \\ \Pi & \text { pressure ratio } \\ \rho & \text { density }\left(\mathrm{kg} / \mathrm{m}^{3}\right) \\ \tau & \text { time constant }(\mathrm{s}) \\ \Phi_{\mathrm{c}} & \text { volumetric flow coefficient } \\ \Psi_{\mathrm{c}} & \text { energy transfer coefficient } \\ \omega & \text { rotational speed (rad/s) }\end{array}$

\section{Subscripts}

$\begin{array}{ll}\text { a } & \text { air } \\ \text { amb } & \text { ambient } \\ \text { c } & \text { compressor } \\ \text { d } & \text { displaced } \\ \text { e } & \text { exhaust } \\ \text { egr } & \text { exhaust gas recirculation } \\ \text { ei } & \text { engine cylinder in } \\ \text { em } & \text { exhaust manifold } \\ \text { eo } & \text { engine cylinder out } \\ \text { f } & \text { fuel } \\ \text { fric } & \text { friction } \\ \text { ig } & \text { indicated gross } \\ \text { im } & \text { intake manifold } \\ \text { m } & \text { mechanical } \\ \text { t } & \text { turbine } \\ \text { tc } & \text { turbocharger } \\ \text { vgt } & \text { variable-geometry turbocharger } \\ \text { vol } & \text { volumetric } \\ \delta & \text { fuel injection }\end{array}$

\section{APPENDIX 2}

\section{Measured variables}

The variables that were measured during stationary measurements can be seen in Table 5. The EGR fraction is calculated by measuring the carbon dioxide concentration in the intake and exhaust manifolds. The variables that were measured during dynamic measurements can be seen in Table 6 .

\section{Sensor-time constants and system dynamics}

To justify the fact that the model captures the system dynamics and not the sensor dynamics, the dynamics of the sensors are analysed and compared with the dynamics seen in the measurements. The time constants of the sensors for the measured outputs during dynamic measurements are shown in Table 6. These time constants are based on sensor data sheets, except for the time constant for the engine torque sensor that is calculated from
Table 5 Measured variables during stationary measurements

\begin{tabular}{lll}
\hline Variable & Description & Unit \\
\hline$M_{\mathrm{e}}$ & Engine torque & $\mathrm{Nm}$ \\
$n_{\mathrm{e}}$ & Rotational engine speed & $\mathrm{r} / \mathrm{min}$ \\
$n_{\mathrm{t}}$ & Rotational turbine speed & $\mathrm{r} / \mathrm{min}$ \\
$p_{\mathrm{amb}}$ & Ambient pressure & $\mathrm{Pa}$ \\
$p_{\mathrm{c}}$ & Pressure after the compressor & $\mathrm{Pa}$ \\
$p_{\mathrm{em}}$ & Exhaust manifold pressure & $\mathrm{Pa}$ \\
$p_{\mathrm{im}}$ & Intake manifold pressure & $\mathrm{Pa}$ \\
$T_{\mathrm{amb}}$ & Ambient temperature & $\mathrm{K}$ \\
$T_{\mathrm{c}}$ & Temperature after the compressor & $\mathrm{K}$ \\
$T_{\mathrm{em}}$ & Exhaust manifold temperature & $\mathrm{K}$ \\
$T_{\mathrm{im}}$ & Intake manifold temperature & $\mathrm{K}$ \\
$T_{\mathrm{t}}$ & Temperature after the turbine & $\mathrm{K}$ \\
$u_{\mathrm{egr}}$ & EGR control signal. 0 - closed; $100-$ open & $\%$ \\
$u_{\mathrm{vgt}}$ & VGT control signal. 0 - closed; $100-$ open & $\%$ \\
$u_{\delta}$ & Injected amount of fuel & $\mathrm{mg} / \mathrm{cycle}$ \\
$W_{\mathrm{c}}$ & Compressor mass flow & $\mathrm{kg} / \mathrm{s}$ \\
$x_{\mathrm{egr}}$ & EGR fraction & - \\
\hline
\end{tabular}

Table 6 Measured variables during dynamic measurements and maximum sensor time constants $\tau_{\max }$

\begin{tabular}{|c|c|c|c|}
\hline Variable & Description & Unit & $\tau_{\max }(\mathrm{ms})$ \\
\hline$n_{\mathrm{t}}$ & Rotational turbine speed & $\mathrm{r} / \mathrm{min}$ & 6 \\
\hline$p_{\mathrm{em}}$ & Exhaust manifold pressure & $\mathrm{Pa}$ & 20 \\
\hline$p_{\mathrm{im}}$ & Intake manifold pressure & $\mathrm{Pa}$ & 15 \\
\hline$W_{\mathrm{c}}$ & Compressor mass flow & $\mathrm{kg} / \mathrm{s}$ & 20 \\
\hline$\tilde{u}_{\text {egr }}$ & $\begin{array}{l}\text { EGR position } \\
0 \text { - closed; } 100 \text { - open }\end{array}$ & $\%$ & $\ll 50$ \\
\hline$\tilde{u}_{\mathrm{vgt}}$ & $\begin{array}{l}\text { VGT position } \\
0 \text { - closed; } 100-\text { open }\end{array}$ & $\%$ & $\gg 25$ \\
\hline$M_{\mathrm{e}}$ & Engine torque & $\mathrm{Nm}$ & 1000 \\
\hline$n_{\mathrm{e}}$ & Rotational engine speed & $\mathrm{r} / \mathrm{min}$ & 26 \\
\hline$u_{\text {egr }}$ & $\begin{array}{l}\text { EGR control signal } \\
0 \text { - closed; } 100 \text { - open }\end{array}$ & $\%$ & - \\
\hline$u_{\mathrm{vgt}}$ & $\begin{array}{l}\text { VGT control signal } \\
0 \text { - closed; } 100 \text { - open }\end{array}$ & $\%$ & - \\
\hline$u_{\delta}$ & Injected amount of fuel & $\mathrm{mg} /$ cycle & - \\
\hline
\end{tabular}

dynamic measurements according to section 8.1. The time constants of the sensors for $n_{\mathrm{t}}, p_{\mathrm{em}}, p_{\mathrm{im}}$ and $W_{\mathrm{c}}$ are significantly faster than the dynamics seen in the measurements in Figs 8 to 10, and these sensor dynamics are therefore neglected. The time constants for the EGR and VGT position sensors are significantly faster than the actuator dynamics and these sensor dynamics are therefore neglected. The time constant for the engine torque sensor is large and it is therefore considered in the validation. However, this time constant is not considered in the proposed model because the model will be used for gas flow control and not for engine torque feedback control. Finally, the sensor dynamics for the engine speed does not effect the dynamic validation results since the engine speed is an input to the 
model and it is also constant in all measurements used here.

\section{APPENDIX 3}

\section{Notes on the contributed model}

A MATLAB Simulink implementation of the model together with a complete parameter file is available from reference [5] or by contacting the authors. The intention of this implementation is to give researchers and engineers access to a validated model that can be used for simulation, development, and verification of new control systems. Researchers working on methodology development can use this model as a challenging reference case since it describes a highly non-linear system with nonminimum phase behaviour.

Together with the implementation and parameter files there is also a MATLAB script runSimEngine.m that simulates the implementation for the two steps in VGT position shown in Figs 11 and 12, and generates these two figures for the eighth-order model. This MATLAB-script illustrates how the implementation can be simulated and gives examples on two step responses that have non-minimum phase behaviour, overshoot, and sign reversal. 